\title{
Determinants of Dirac Boundary Value Problems over Odd-Dimensional Manifolds
}

\author{
S.G. Scott $^{1}$ \\ Departamento de Matématicas, Universidad de los Andes, Bogotá A.A.4976, Colombia
}

Received: 25 October 1993/in revised form: 27 January 1995

\begin{abstract}
We present a canonical construction of the determinant of an elliptic selfadjoint boundary value problem for the Dirac operator $D$ over an odd-dimensional manifold. For 1-dimensional manifolds we prove that this coincides with the $\zeta$-function determinant. This is based on a result that elliptic self-adjoint boundary conditions for $D$ are parameterized by a preferred class of unitary isomorphisms between the spaces of boundary chiral spinor fields. With respect to a decomposition $S^{1}=X^{0} \cup X^{1}$, we explain how the determinant of a Dirac-type operator over $S^{1}$ is related to the determinants of the corresponding boundary value problems over $X^{0}$ and $X^{1}$.
\end{abstract}

\section{Introduction}

Let $X$ be a compact odd-dimensional Riemmanian spin manifold with boundary $Y$. We assume there is a collar neighbourhood $U=[0,1] \times Y$ of the boundary in which the Riemannian metric is a product metric. Fix a choice of spin structure, and let $S$ be the complex spinor bundle over $X$. The Dirac operator $D: C^{\infty}(X ; S) \rightarrow C^{\infty}(X ; S)$ is the first-order elliptic differential operator defined at $x \in X$ by $D s=\sum_{1} e_{i} \cdot \nabla_{e_{i}} s$, where $\nabla$ is the canonical metric connection on $S$ and $\left\{e_{l}\right\}$ is an orthonormal frame for $T_{x} X$. The $e_{i}$ act on $S$ by Clifford multiplication. The restriction of $S$ to $Y$ may be identified with the spinor bundle over $Y$ with $Z_{2}$ grading $S_{Y}=S^{+} \oplus S^{-}$. That induces a decomposition of the boundary spinor fields $F=F^{+} \oplus F^{-}$into positive and negative chirality with respect to which the Dirac operator $D_{Y}$ over the boundary splits into the chiral operator $D_{Y}^{+}: F^{+} \rightarrow F^{-}$, whose index is calculated by evaluating the $\hat{A}$-cohomology class over $Y$, and its formal adjoint $D_{Y}^{-}$. We assume that $D_{Y}$ is invertible.

By a boundary value problem $D_{W}$ for $D$, we shall mean $D$ with restricted domain $C_{W}^{\infty}(X ; S)=\left\{\psi \in C^{\infty}(X ; S): P_{W} b \psi=0\right\}$, where $P_{W}: C^{\infty}(Y ; S) \rightarrow C^{\infty}(Y ; S)$ is a pseudodifferential projection operator (of order 0 ) with range $W$, and $b: C^{\infty}(X ; S)$

${ }^{1}$ Current address: Physics Department, Oxford University, Oxford, OX1 3PU, U.K. 
$\rightarrow C^{\infty}\left(Y ; S_{Y}\right)$ is the operator restricting sections to the boundary. We shall refer to $W$ as a boundary condition for $D$.

The purpose of this paper is to present a construction of determinants of elliptic self-adjoint boundary value problems for $D$ using the following theorem.

Theorem 1.1. There is a canonical one-to-one correspondence between elliptic selfadjoint boundary conditions for the Dirac operator over an odd-dimensional spin manifold and $L^{2}$-unitary isomorphisms $g: F^{+} \rightarrow F^{-}$between the positive and negative boundary spinor fields which differ from $g_{+}=i\left(D_{Y}^{+} D_{Y}^{-}\right)^{-1 / 2} D_{Y}^{+}: F^{+} \rightarrow F^{-}$ by a smoothing operator.

In [4] the basic unitary isomorphism $g_{+}$is considered in the context of the index theorem for families for odd-dimensional manifolds with boundary.

A boundary condition $W$ for $D$ is referred to as elliptic if it lies in a certain infinite-dimensional Grassmannian $G r$ associated to the space of boundary spinor fields. Roughly this is the requirement that we only consider those boundary conditions which are commensurable with the Atiyah-Patodi-Singer boundary condition which, for even-dimensional $X$, was studied in detail in [3] and the index "defect" identified as essentially the $\eta$-invariant of the boundary Dirac operator. To justify the use of the term elliptic we give in Appendix A of the paper a construction of a specific parametrix for $D_{W}$, from which it follows that $D_{W}$ has the principal analytic properties of an elliptic operator over a closed manifold. We refer to [8] for detailed background on elliptic boundary value problems for Dirac operators. The elliptic boundary conditions considered here form a dense subset of those studied in $[7,8,10]$.

We refer to the determinant of $D_{W}$ constructed using Theorem (1.1) as the canonical determinant and we denote it by $\operatorname{det}_{\mathscr{C}} D_{W}$. Specifically, if $K$ is the restriction to the boundary of the space of harmonic spinors $\operatorname{Ker} D$, then $K$ is a self-adjoint boundary condition for $D$ and one has

Theorem 1.2. Let $W$ be a self-adjoint boundary condition for $D$. If $D_{W}$ is invertible, there is a canonical identification

$$
\operatorname{det}_{\mathscr{C}} D_{W}=\operatorname{det} \frac{1}{2}\left(1-g_{0} h\right)
$$

where $W$ and $K$ are respectively the graphs of the unitary isomorphisms $g: F^{+} \rightarrow$ $F^{-}$and $h: F^{+} \rightarrow F^{-}$from Theorem (1.1), and $g_{0}=-g^{-1}$.

Here the right-hand side denotes the usual determinant as a number in $C$ of an operator of the form $1+t$, where $t: F^{+} \rightarrow F^{+}$is a trace-class for the $L^{2}$ norm [27], defined by $\operatorname{det}(1+t)=\sum_{k=0}^{\infty} \operatorname{Tr}\left(\wedge^{k} t\right)$.

A more enlightening way to view Theorem (1.1) and Theorem (1.2) is as follows. In the seminal paper of Quillen [18] it was explained that the determinant associated to a smooth family $\mathscr{A}$ of Dirac operators arises not as a function $\mathscr{A} \rightarrow C$ but rather as a section of a complex line bundle $L$ over $\mathscr{A}$; the so-called determinant line bundle. Consequently the obstruction to writing the determinant as a globally defined function on $\mathscr{A}$ is precisely the obstruction to finding a global trivialization of $L$. If that obstruction vanishes one then looks for a canonical choice of trivialization that naturally extends the theory of finite-dimensional determinants. In [18] that is achieved for a family of $\bar{\partial}$-operators on a Hermitian vector bundle over a closed Riemann surface by defining a flat connection on the determinant line bundle using a construction of $\zeta$-function determinants of Laplacians. This procedure 
defines a natural trivialization of $L$ and hence an identification of the determinant section as a function up to a phase ambiguity, and this has come to be accepted as essentially the canonical method for calculating such determinants. (In [5] the $\zeta$-function metric was constructed for the determinant line bundle associated to a general family of Dirac operators over a closed manifold and the curvature, representing the first Chern class of the bundle, identified as the 2-form component of the local family's index theorem.)

In this general context, one may view the Grassmannian $\mathrm{Gr}$ as the parameter space $\mathscr{A}$ of a smooth family of Dirac boundary value problems. Over $G r$ one still has the Quillen determinant line bundle $L$ with canonical section det : $G r \rightarrow L$ which takes $W$ to det $D_{W}$. In fact, the holomorphic line bundle $L$ is isomorphic to the determinant line bundle of [17] which has non-zero first Chern class and hence is topologically non-trivial, and so no global trivialization of $L$ exists. However, over the component $G r_{\text {sso }}$ (the isotropic Grassmannian) of the restricted Grassmannian parameterizing self-adjoint boundary conditions for $D$ the determinant bundle $L$ is canonically trivial. Indeed, that is the content of Theorem (1.1) which defines a trivialization $\sigma: G r_{\text {so }} \rightarrow L$, and from Theorem (1.2),

$$
\operatorname{det} D_{W}=\operatorname{det} \frac{1}{2}\left(1-g_{0} h\right) \cdot \sigma(W) \text {. }
$$

The determinant line bundle is discussed in Sect. 4 of this paper.

The proof of Theorem (1.2) is straightforward. The harder step comes in the identification of the canonical determinant with the $\zeta$-function determinant $\operatorname{det}_{\zeta} D_{W}$. That determinant is defined via the $\zeta$-function norm $\|\cdot\|_{\zeta}$ defined on the determinant line of $D_{W}$ and formally $\left|\operatorname{det}_{\zeta} D_{W}\right|=\left\|\operatorname{det} D_{W}\right\|_{\zeta}$. In general though, it is not clear that $\operatorname{det}_{\zeta} D_{W}$ is defined for the same analytic reasons that compel one in the case of closed manifolds to consider the $\zeta$-function determinant of the Laplacian rather than the operator itself; that is the origin of the phase ambiguity. When $X$ is one dimensional, however, and $D$ is a Dirac-type operator of the form $i \nabla_{d / d x}$ acting on the sections of a (trivial) $U(n)$-bundle $\mathscr{E}$ with unitary connection $\nabla$, the $\zeta$-function determinant can be defined directly. We take $X=[0,1]$ and denote the boundary fibres of $\mathscr{E}$ by $\mathscr{E}_{0}, \mathscr{E}_{1}$.

Theorem 1.3. Let $D_{W}$ be invertible. Then $\zeta_{D_{W}}(s)=\operatorname{Tr} D_{W}^{-s}$ is well-defined for $\operatorname{Re} s>1$ and has an analytic continuation to all of $C$. The $\zeta$-function determinant exists and

$$
\operatorname{det}_{\zeta} D_{W}=\operatorname{det}\left(1-g_{0} h\right),
$$

where $W$ and $K$ are respectively the graphs of the unitary isomorphisms $g: \mathscr{E}_{0} \rightarrow$ $\mathscr{E}_{1}$ and $h: \mathscr{E}_{0} \rightarrow \mathscr{E}_{1}$ from Theorem (1.1) and $g_{0}=-g^{-1}$. The isomorphism $h$ is the parallel transport of the connection $\nabla$.

Relative to a trivialization of $\mathscr{E}$ the isomorphisms $g, h$ are identified as elements of the unitary group $U(n)$, changing the trivialization only changes $g_{0} h$ by conjugation by an element of $U(n)$ and hence the right-hand side of (2) is unambiguously defined as a complex number. Theorem (1.3) is complementary to the work of [9] and [11] on determinants in 1 dimension.

The existence of the canonical trivialization $\sigma$ of $L_{\mid G r_{\text {iso }}}$, in addition to the usual $\zeta$-function trivialization, is because of the extra degree of freedom introduced by the choice of boundary condition, and we exploit this fact repeatedly in our constructions. One effect of this extra degree of freedom is that there is something of a 
menagerie of different but isomorphic determinant line bundles over. $G r$. In particular, in Sect. 5c we use this to give a third distinct construction of the determinant for $\operatorname{dim} X=1$ which coincides with the $\zeta$-function and canonical determinants up to a factor of $i^{n}$.

Having established these identifications it is natural to ask if there is a relation with determinants over closed manifolds. To answer that, consider the closed double $M=X \cup_{Y} X^{-}$, where $X^{-}$is a copy of $X$ with reverse orientation, which by reflection has a Riemannian metric equal to a product in a tubular neighbourhood $V=[-1,1] \times Y$ of $Y=\{0\} \times Y$. Over $M$ is the double spinor bundle $S_{M}$ formed by gluing together two copies of the spinor bundle $S$ via the automorphism $\sigma: S_{Y} \rightarrow S_{Y}$. A section $\psi_{M} \in C^{\infty}\left(M ; S_{M}\right)$ then consists of a pair of sections $\left(\psi, \psi^{-}\right) \in C^{\infty}(X ; S) \oplus C^{\infty}\left(X^{-} ; S\right)$ such that at $\{0\} \times Y$ the sections $\sigma \psi$ and $\psi^{-}$ have the same values and normal derivatives of all orders. One then has the firstorder elliptic "Dirac" operator $D_{M}=D \cup(-D): C^{\infty}\left(M ; S_{M}\right) \rightarrow C^{\infty}\left(M ; S_{M}\right)$, defined by $D_{M}\left(\psi, \psi^{-}\right)=\left(D \psi,-D \psi^{-}\right)$. More generally, given Riemannian spin manifolds $X^{0}, X^{1}$ with the same boundary $Y$, up to orientation, and with spinor bundles $S^{0}, S^{1}$ such that the restricted spinor bundles $S_{Y}^{0}, S_{Y}^{1}$ coincide and all topological and geometric data agree at $Y$, one may form the corresponding elliptic operator $D_{M}=D^{0} \cup-D^{1}$ over $M=X^{0} \cup_{Y} X^{1}$. The canonical determinant of $D_{M}$ is defined and we denote it by $\operatorname{det}_{\mathscr{C}} D_{M}$. We denote by $K^{0}$ and $K^{1}$ the respective restrictions to the boundary of the space of harmonic spinors of $D^{0}$ and $D^{1}$.

Theorem 1.4. Let $M$ be odd-dimensional. If $D_{M}$ is invertible, there is a canonical identification

$$
\operatorname{det}_{\mathscr{C}} D_{M}=\operatorname{det} \frac{1}{2}\left(1-h_{1} h_{0}\right)
$$

where $K^{0}$ and $K^{1}$ are respectively the graphs of the unitary isomorphisms $h_{0}$ : $F^{+} \rightarrow F^{-}$and $h_{1}: F^{-} \rightarrow F^{+}$from Theorem (1.1).

In dimension 1, with $S^{1}=X^{0} \cup X^{1}$, the isomorphisms $h_{0}$ and $h_{1}$ represent the parallel transport along $X^{0}$ and $X^{1}$ with respect to a unitary connection $\nabla_{d / d x}$ : $C^{\infty}\left(S^{1} ; \mathscr{E}_{S^{1}}\right) \rightarrow C^{\infty}\left(S^{1} ; \mathscr{E}_{S^{1}}\right)$ on a Hermitian $n$-bundle $\mathscr{E}_{S^{1}}$ over $S^{1}$. Hence, since $h_{1} h_{0}$ is the holonomy of $\nabla$ around $S^{1}$ (and since the factor of $1 / 2$ on the right-hand side of (3) can be removed when $\operatorname{dim} X=1$ ), then $\operatorname{det}_{\mathscr{\zeta}} D_{S^{1}}$, where $D_{S^{1}}=i \nabla_{d / d x}$, coincides with the well-known value of the $\zeta$-function determinant $[1,9]$.

For $\operatorname{dim} X=1$ Theorem 1.3 and Theorem 1.4 are related as follows. Let $\mathscr{E}^{i}=$ $\mathscr{E}_{S^{1} \mid X^{i}}$ and let $D^{i}$ be the restriction of $D_{S^{1}}$ to $C^{\infty}\left(X^{i} ; \mathscr{E}\right)$.

\section{Theorem 1.5.}

$$
\operatorname{det}_{\mathscr{C}} D_{S^{1}}=\int_{U(n)} \operatorname{det}_{\mathscr{C}} D_{W}^{0} \operatorname{det}_{\mathscr{C}} D_{W^{\perp}}^{1} d W
$$

The integral in (4) is carried out over the unitary group under the isomorphism $U(n) \cong G r_{\text {iso }}$ defined by Theorem (1.1). Thus Theorem (1.5) states that the determinant over the closed manifold is obtained by integrating away the choice of self-adjoint boundary condition in the determinants over the two halves. We could of course have written (4) in terms of $\zeta$-function determinants, the difference is purely notational. An open question is whether (4) may indicate a relation between the $\zeta$-function metric on the determinant line bundle for a general family of Dirac boundary value problems and the $\zeta$-function metric for the corresponding family of Dirac operators over the closed double manifold. 
Since the topic of determinants of boundary value problems has taken on a specific interest in mathematical physics with the development of topological quantum field theories [2,26], we conclude this paper with some brief comments on the relation between Theorems (1.1)-(1.5) and $0+1$-dimensional TQFT. For a specific account of the relation of conformal field theory to Grassmannians and elliptic boundary value problems for $\bar{\partial}$-operators over a Riemann surface we refer to $[25,28]$.

\section{A Grassmannian of Dirac Boundary Value Problems}

In this section we describe the analytic constructions in more detail. Let $X$ be a compact spin manifold with boundary $Y$. Then the Dirac operator $D$ over $X$ is formally self-adjoint with respect to the $L^{2}$-Hermitian inner-product

$$
\left\langle\psi_{1}, \psi_{2}\right\rangle_{S}=\int_{X}\left(\psi_{1}, \psi_{2}\right)_{S} d x
$$

where $d x$ denotes the Riemannian measure on $X$. This means that for all $\psi_{1}, \psi_{2} \in$ $C^{\infty}(X ; S)$ with supports disjoint from the boundary of $X$ one has

$$
\left\langle D \psi_{1}, \psi_{2}\right\rangle_{S}=\left\langle\psi_{1}, D \psi_{2}\right\rangle_{S}
$$

In the collar neighbourhood $U=[0,1] \times Y$ of the boundary $Y \cong\{0\} \times Y$ we choose a Riemannian metric $g$ on $X$ which splits isometrically as $g_{U}=d u^{2}+g_{Y}$, where $u$ is the normal coordinate to the boundary and $g_{Y}$ the induced metric on $Y$. Over $U$ the Dirac operator has the form

$$
D_{\mid U}=\sigma\left(\frac{\partial}{\partial u}+A\right),
$$

where the symbol map $\sigma=\sigma(D)(d u): S_{Y} \rightarrow S_{Y}$ is the bundle isomorphism given by Clifford multiplication by the inward unit normal $d u$ in $T^{*} U$. We note that $\sigma^{2}=-1$ and that $\sigma$ is an isometry with respect to the induced inner-product $\langle$,$\rangle on$ $C^{\infty}\left(Y ; S_{Y}\right)$. The boundary operator $A=D_{Y} \sigma: C^{\infty}\left(Y ; S_{Y}\right) \rightarrow C^{\infty}\left(Y ; S_{Y}\right)$ is a selfadjoint first-order elliptic differential operator independent of the normal coordinate $u$. Because $Y$ is a closed manifold, $A$ has a real and discrete spectrum $\lambda$ with smooth eigenvectors $\phi_{i}$. Because $D$ is formally self-adjoint the following equalities hold,

$$
\sigma^{*}=-\sigma \quad \sigma A+A \sigma=0,
$$

so that $A$ is of degree 1 with respect to the mod 2 grading defined by $\sigma$.

The Grassmannian of elliptic boundary conditions is defined with respect to the energy polarization $F=H^{+} \oplus H^{-}$of the space of boundary spinor fields, where the subspaces $H^{+}$and $H^{-}$are spanned, respectively, by those eigenvectors of $A$ with non-negative and negative eigenvalues. The polarization is given by an involution $J: F \rightarrow F$ equal to +1 on $H^{+}$and -1 on $H^{-}$, which defines canonical pseudodifferential projections

$$
P^{ \pm}=\frac{1}{2}(I \pm J): F \rightarrow H^{ \pm}
$$

One thus obtains the preferred boundary value problem $D_{H^{+}}: C_{H^{+}}^{\infty}(X ; S) \rightarrow C^{\infty}$ $(X ; S)$ studied in [3]. 
Definition. The restricted Grassmannian $\mathrm{Gr}$ is the set of all closed subspaces $W$ of $F$ such that $W=\operatorname{Ker}\left(J_{W}-1\right)$, where $J_{W}: F \rightarrow F$ is a formally self-adjoint involution $\left(J_{W}^{2}=1\right)$ such that $J_{W}-J$ is a smoothing operator.

For each $W$ in $G r$ the involution $J_{W}$ defines (with respect to $L^{2}\left(Y ; S_{Y}\right)$ ) formally self-adjoint orthogonal projections $P_{W}: F \rightarrow W$ and $P_{W^{\perp}}: F \rightarrow W^{\perp} \stackrel{\text { def }}{=} \bar{W}^{\perp} \cap F$. Hence, as a corollary to Lemma (2.1), the restricted Grassmannian is a parameter space of boundary value problems for $D$. We refer to $D_{W}$ for $W \in G r$ as a Dirac boundary value problem.

Lemma 2.1. For $W \in G r$ the projection $P_{W}$ (resp. $P_{W \perp}$ ) is a pseudodifferential operator of order 0 with leading symbol $\sigma\left(P_{W}\right)(y, \zeta): S_{y} \rightarrow S_{y}, y \in Y$, (resp. $\left.\sigma\left(P_{W \perp}\right)(y, \zeta)\right)$, given by the projection onto the eigenspaces of $-\sigma(A)(y, \zeta)$ with eigenvalues having positive (resp. negative) imaginary part.

Proof. Because the leading symbol of $P^{+}$has symbol as stated, the generalisation to all $W \in G r$ is the observation that $P_{W}-P^{+}=\frac{1}{2}\left(J-J_{W}\right)$, which is a smoothing operator, and consequently that $\sigma\left(P_{W}-P^{+}\right)(y, \zeta)=0$.

We denote by $\mathrm{Gr}^{-}$the opposite restricted Grassmannian defined by replacing $J_{W}$ by $-J_{W}$, thus reversing the roles of $H^{+}$and $H^{-}$. It is immediate from the definition of $G r$ that if $W \in G r$ then $W^{\perp} \in G r^{-}$. We also note that if $W_{0}, W_{1} \in G r$ then $P_{W_{1}} \circ$ $i_{W_{0}}: W_{0} \rightarrow W_{1}$ is a Fredholm operator, and that $P_{W_{1}^{\perp}} \circ i_{W_{0}}=S_{\mid W_{0}}$, where $S: F \rightarrow F$ is a smoothing operator. (We recall that an operator on a Frechet space is Fredholm if and only if it is invertible modulo compact operators, and that is equivalent to the assertion that the operator has closed range and finite-dimensional kernel and cokernel.) For a detailed account of the properties of restricted Grassmannians we refer to $[8,17$ and 25].

Two boundary value problems $D_{W}: C_{W}^{\infty}(X ; S) \rightarrow C^{\infty}(X ; S)$ and $D_{W^{*}}: C_{W^{*}}^{\infty}$ $(X ; S) \rightarrow C^{\infty}(X ; S)$ are formally adjoint if the domain $C_{W^{*}}^{\infty}(X ; S)$ of $D_{W^{*}}$ consists of those $\eta \in C^{\infty}(X ; S)$ such that $\left\langle D_{W} \psi, \eta\right\rangle_{S}=\langle\psi, D \eta\rangle_{S}$ for all $\psi \in C_{W}^{\infty}(X ; S)$. In the case that $W=W^{*}$ we refer to $D_{W}$ as a self-adjoint boundary value problem and to $W$ as a self-adjoint boundary condition. The following characterization of self-adjoint boundary conditions is well known [7].

Lemma 2.2. $W \in G r$ is a self-adjoint boundary condition for $D$ if and only if $W$ is in the isotropic Grassmannian $G r_{\text {iso, }}$, defined to be the real submanifold of the restricted Grassmannian Gr parameterizing subspaces $W$ of $F$ maximal isotropic for the bilinear form

$$
\beta\left(\phi_{0}, \phi_{1}\right)=\int_{Y}\left(\sigma \phi_{0}, \phi_{1}\right) d y .
$$

Equivalently, $W$ is self-adjoint if and only if

$$
\sigma P_{W}+P_{W} \sigma=\sigma .
$$

Proof. The first statement follows immediately from Green's formula

$$
\left\langle D \psi_{0}, \psi_{1}\right\rangle_{S}-\left\langle\psi_{0}, D \psi_{1}\right\rangle_{S}=\int_{Y}\left(\sigma b \psi_{0}, b \psi_{1}\right) d y,
$$

for $\psi_{0}, \psi_{1} \in C^{\infty}(X ; S)$. The maximality requirement ensures that the unbounded operator $D_{W}$ is self-adjoint and not just symmetric. Further, if $\psi_{0} \in C_{W}^{\infty}(X ; S)$ then $\beta\left(b \psi_{0}, b \psi_{1}\right)=\int_{Y}\left\langle\sigma b \psi_{0},-\sigma\left(I-P_{W}\right) \sigma b \psi_{1}\right\rangle$, which implies the second statement. 
In Appendix A we construct a parametrix for an elliptic Dirac boundary value problem $D_{W}, W \in G r$, from which it follows that $D_{W}$ is Fredholm and that if $W$ is a self adjoint boundary condition then $D_{W}$ is essentially self-adjoint, that is, $D_{W}$ has a unique $L^{2}$ self-adjoint extension. That implies the spectral theorem.

Proposition 2.1. Let $W \in G r$ be a self-adjoint boundary condition for $D: C^{\infty}$ $(X ; S) \rightarrow C^{\infty}(X ; S)$. Then there is a direct sum decomposition of $L^{2}(X ; S)$ into finite dimensional subspaces each of which consists of smooth sections and is an eigenspace for $D_{W}$. The eigenvalues $\lambda$ of $D_{W}$ are real and discrete.

This theorem and its analogue for the Laplacian (which are proved more generally in [8]) combined with the existence of the parametrix are needed to define the $\zeta$-function for a Dirac boundary value problem.

The crucial property of the restricted Grassmannian is the following.

Proposition 2.2. The space $K=b(\operatorname{ker} D)$ of harmonic spinors of $D$ is in $G r$.

We refer to $K$ as the space of harmonic spinors of $D$ in the sense of

Lemma 2.3. The restriction map $b_{\mid \operatorname{ker} D}: \operatorname{ker} D \rightarrow K$ is bijective.

Proof. First let us recall the theorem that $D$ satisfies the unique continuation property, which means that any solution $\psi$ of the equation $D \psi=0$ that vanishes on an open subset of $X$ vanishes on the whole manifold [8]. Now let $C_{0}^{\infty}(X ; S)=\{\psi \in$ $\left.C^{\infty}(X ; S): b \psi=0\right\}$ and let $D_{0}$ denote the restriction of $D$ to $C_{0}^{\infty}(X ; S)$. Then the statement of the lemma is equivalent to the statement that $D_{0}$ is injective. To see that $D_{0}$ is injective, expand $\psi \in C_{0}^{\infty}(X ; S)$ in the collar $U$ as $\psi(u, y)=\sum_{\lambda} \psi_{\lambda}(u) \phi_{\lambda}(y)$ and notice that $D_{0} \psi=0$ implies $\psi_{\lambda}(u)=e^{-i u} \psi_{\lambda}(0)$. Because $\psi_{\lambda}(0)=0$ an appeal to the unique continuation property proves the lemma.

Proof of Proposition (2.2). From [16] (chapter XVII) the pseudodifferential projection $P_{K}: F \rightarrow K$ has leading symbol equal to the leading symbol of $P^{+}\left(P_{K}\right.$ is called the Calderon projector). Thus $\sigma\left(P^{+}\right)=\sigma\left(P_{K}\right)$ and by symbolic calculus $\sigma\left(P^{+}-P_{K}\right)=0$, which implies that $P_{K}=P^{+}+s$ for some pseudodifferential operator $s: F \rightarrow F$ of order -1 . In particular, $s$ is a compact operator and so the projection $p r_{+}: K \rightarrow H^{+}$is Fredholm and the projection $p r_{-}: K \rightarrow H^{-}$is compact. Hence, since $K$ has virtual dimension zero, by the argument of [17](p.103) $K$ is the graph of a compact operator $H^{+} \rightarrow H^{-}$. The assertion that $K$ has virtual dimension zero is the assertion that ind $p r_{+}=0$, where ind $p r_{+}=\operatorname{dimKer} p r_{+}-\operatorname{dimCoker} p r_{+}$ is the index of $p r_{+}$. To prove that we define

$$
\left(D, H^{+}\right): C^{\infty}(X ; S) \rightarrow C^{\infty}(X ; S) \oplus H^{+}, \quad \psi \mapsto\left(D \psi, P^{+} b \psi\right)
$$

and note that the diagram of maps

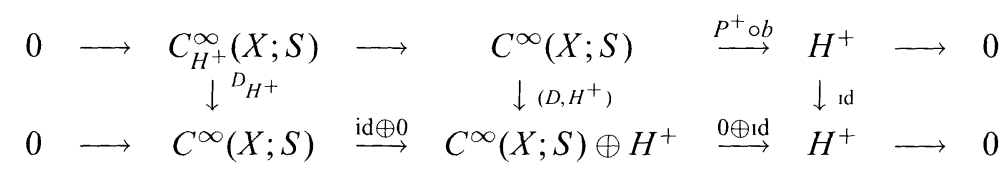

is commutative with exact rows. Hence, since $\operatorname{Ker}(\mathrm{id})=\operatorname{Coker}(\mathrm{id})=0$, there are isomorphisms $\operatorname{Ker} D_{H^{+}} \cong \operatorname{Ker}\left(D, H^{+}\right)$and $\operatorname{Coker} D_{H^{+}} \cong \operatorname{Coker}\left(D, H^{+}\right)[6]($ p.7). Since $D_{H^{+}}$is Fredholm so is $\left(D, H^{+}\right)$, and ind $D_{H^{+}}=\operatorname{ind}\left(D, H^{+}\right)$. From the 
commutative diagram with exact rows

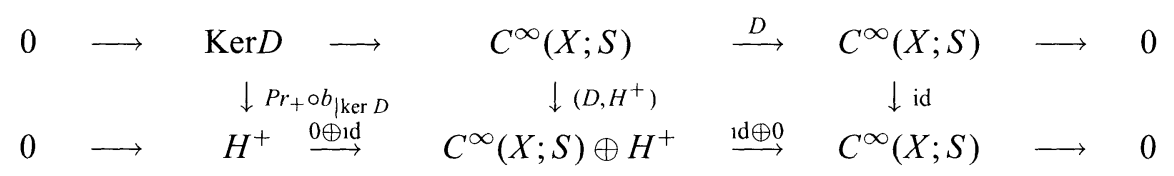

we have similarly, ind $\left(D, H^{+}\right)=$ind $p r_{+} \circ b_{\mid \mathrm{ker} D}=$ ind $p r_{+}+$ind $b_{\mid k e r D}=$ ind $p r_{+}$, the last equality coming from Lemma (2.3). But because $D_{Y}$ is invertible then $D_{H}+$ is self-adjoint, and hence ind $D_{H^{+}}=0$, which proves the assertion. (That $D_{H^{+}}$is self-adjoint is well-known, see [3,8] and for odd-dimensional $X$ see also Theorem (1.1).)

To show that $K \in G r$ it is enough to show $K$ is actually the graph of a smoothing operator $T: H^{+} \rightarrow H^{-}$. To see that, we use the product structure $U=[0,1] \times Y$. Let $D^{\prime}$ denote the operator defined on the manifold $X \backslash U$ with boundary $Y$ so that $D$ is $D^{\prime}$ extended by $\sigma\left(\frac{\partial}{\partial u}+A\right)$ over $U$. Then by the above argument the space of harmonic spinors $K^{\prime}$ of $D^{\prime}$ occurs as the graph of some compact operator $C$ : $H^{+} \rightarrow H^{-}$. For $\psi \in K$ there is a unique $\psi_{0} \in K^{\prime}$ that interpolates across the collar through the monodromy of the elliptic differential operator $(-\partial / \partial u+A)$, where we reparameterize $U$ by replacing $u$ by $1-u$. With respect to the energy polarisation $F=H^{+} \oplus H^{-}$we may write $\psi_{0}=\left(\psi_{+}(0), \psi_{-}(0)\right), \psi=\left(\psi_{+}(1), \psi_{-}(1)\right)$, and $A=$ $A^{+} \oplus A^{-}$, so that

$$
\psi_{-}(1)=e^{A^{-}} \psi_{-}(0)=e^{A^{-}} C \psi_{+}(0)=e^{A^{-}} C e^{-A^{+}} \psi_{+}(1)
$$

Hence $K$ arises as the graph of the operator $S=e^{A^{-}} C e^{-A^{+}}: H^{+} \rightarrow H^{-}$. However,

$$
e^{-A^{+}}=P^{+} e^{-|A|} P^{+} \text {and } e^{A^{-}}=P^{-} e^{-|A|} P^{-},
$$

and since $e^{-|A|}$ has a smooth kernel and $C$ is a compact operator then $S$ is a smoothing operator, and that is what we needed to prove.

\section{Proof of Theorem 1.1}

Let $X$ be an odd-dimensional spin manifold. Then in the collar neighbourhood $U$ of the boundary the Dirac operator has the form

$$
D_{\mid U}=\left(\begin{array}{cc}
i & 0 \\
0 & -i
\end{array}\right)\left(\frac{\partial}{\partial u}+\left(\begin{array}{cc}
0 & -i D_{Y}^{-} \\
i D_{Y}^{+} & 0
\end{array}\right)\right)
$$

where $D_{Y}^{+}: F^{+} \rightarrow F^{-}$is the chiral Dirac operator over $Y$. Notice that one has

$$
S_{Y}^{ \pm}=\left\{v \in S_{Y}: \sigma(v)= \pm i v\right\}
$$

Notice also that we have two distinct canonical polarizations of the space of boundary spinor fields; namely, the splitting of $F$ into positive and negative energy $H^{ \pm}$, and the splitting of $F$ into spinor fields of positive and negative chirality $F^{ \pm}$. These splittings are, however, naturally isomorphic in the following precise sense. 
Since $D_{Y}^{+}$is invertible then $H^{+}$is the graph of the canonical unitary isomorphism

$$
g_{+}=i\left(D_{Y}^{+} D_{Y}^{-}\right)^{-1 / 2} D_{Y}^{+}: F^{+} \rightarrow F^{-},
$$

and we write $H^{+}=\Gamma\left(g_{+}\right)$. The significance of this for the (APS) Dirac boundary value problem $D_{H^{+}}: C_{H^{+}}^{\infty}(X ; S) \rightarrow C^{\infty}(X ; S)$ is that it is then self-adjoint. This observation portrays the more general principle expressed in Theorem (1.1), and that is an immediate consequence of

Proposition 3.1. There is a canonical one-to-one correspondence between subspaces $W$ in $G r_{\text {iso }}$ and $L^{2}$-unitary isomorphisms $g: F^{+} \rightarrow F^{-}$between the boundary chiral spinor fields which differ from $g_{+}$by a smoothing operator. The isotropic Grassmannian $G r_{\text {iso }}$ is precisely the space of graphs of all such unitary isomorphisms $\mathrm{g}$.

Proof. Let us show that $\Gamma(g) \in G r_{\text {iso }}$. With respect to the decomposition $F=F^{+} \oplus$ $F^{-}$the Hermitian $L^{2}$ inner-product splits as $\langle\rangle=,\langle,\rangle^{+}+\langle,\rangle^{-}$, where $\langle,\rangle^{ \pm}$are the $L^{2}$ inner-products on $F^{ \pm}$. For any unitary isomorphism $g: F^{+} \rightarrow F^{-}$one then has $\left\|g \psi^{+}\right\|^{-}=\left\|\psi^{+}\right\|^{+}$for all positive spinor fields $\psi^{+} \in F^{+}$.

The isotropic condition is satisfied because if $\psi_{j} \in \Gamma(g)$, for $j=1,2$, then $\psi_{j}=$ $\left(\psi_{j}^{+}, g \psi_{j}^{+}\right)$for some $\psi_{j}^{+} \in F^{+}$, and hence, since $\sigma=i \oplus(-i)$,

$$
\beta\left(\psi_{1}, \psi_{2} ; D\right)=\left\langle\sigma \psi_{1}, \psi_{2}\right\rangle=\left\langle i \psi_{1}^{+}, \psi_{2}^{+}\right\rangle^{+}+\left\langle-i g \psi_{1}^{+}, g \psi_{2}^{+}\right\rangle^{-}=0,
$$

and so $\Gamma(g)$ is an isotropic subspace of $F$ with respect to $\beta$. In fact (from (8)), to see that it is maximal isotropic it is enough to show that $\sigma P_{\Gamma(g)}+P_{\Gamma(g)} \sigma=\sigma$. To see that, let $I$ denote the identity operator and let $T: F^{+} \rightarrow F^{-}$be the smoothing operator such that $g=g_{+}+T$. Then the orthogonal projection $P_{\Gamma(g)}: F \rightarrow \Gamma(g)$ is given relative to the chiral spinor polarization by

$$
P_{\Gamma(g)}=\frac{1}{2}\left(\begin{array}{cc}
I & g^{*}+T^{*} \\
g+T & I
\end{array}\right)
$$

and the identity follows. Further, the operator $J_{\Gamma(g)}=2 P_{\Gamma(g)}-I: F \rightarrow F$ is a formally self-adjoint involution on $F$ with $\Gamma(g)=\operatorname{Ker}\left(J_{\Gamma(g)}-I\right)$. If $J$ is the involution on $F$ corresponding to $\mathrm{H}^{+}$, then

$$
J_{\Gamma(g)}-J=\left(2 P_{\Gamma(g)}-I\right)-\left(2 P^{+}-I\right)=\left(\begin{array}{cc}
0 & T^{*} \\
T & 0
\end{array}\right),
$$

and so since $T$, and therefore $T^{*}$, is smoothing then so is $J_{\Gamma(g)}-J$. Hence $\Gamma(g)$ is in the restricted Grassmannian $G r$.

Conversely, we assert that each $W \in G r_{\text {iso }}$ arises as the graph of a unitary isomorphism $g: F^{+} \rightarrow F^{-}$which differs from $g_{+}$by a smoothing operator. To see this we use a slightly generalized version of the proof of the cobordism invariance of the analytical index [16]. First, note from (11) that the fibre projections $S_{y} \rightarrow S_{y}^{ \pm}$ for $y \in Y$ are given by $B^{ \pm}(y)=\frac{1}{2}(I \pm i \sigma(y, d u))$, where we write $\sigma(D)(y, d u)=$ $\sigma(y, d u)$, so that

$$
\sigma(y, d u)=i\left(B^{+}(y)-B^{-}(y)\right) \quad \text { and } \quad B^{+}(y)+B^{-}(y)=I: S_{y} \rightarrow S_{y} .
$$


We now define $B^{ \pm}: F \rightarrow F^{ \pm}$by $\left(B^{ \pm} \phi\right)(y)=B^{ \pm}(y) \phi(y)$. Hence

$$
\beta\left(\phi_{1}, \phi_{2} ; D\right)=i \int_{Y}\left(B^{+} \phi_{1}-B^{-} \phi_{1}, \phi_{2}\right) d y \text {. }
$$

Let $W \in G r_{\text {iso }}(F)$. We define a linear $g \in \operatorname{Hom}\left(F^{+}, F^{-}\right)$as follows. If $\phi \in W$, then

$$
g\left(B^{+} \phi\right)=B^{-} \phi .
$$

We claim that $g: F^{+} \rightarrow F^{-}$is a unitary isomorphism.

First we must show that the projection $B^{+}: W \rightarrow F^{+}$is surjective. Define

$$
C: F \rightarrow F, \quad C=B^{+} P_{W}+B^{-} P_{W \perp}
$$

and suppose that $C$ is bijective. Let $\xi \in F^{+}$and write $\eta=C^{-1} i_{+} \xi=C^{-1} \xi$, where $i_{+}: F^{+} \rightarrow F$ denotes the inclusion. Then

$$
\xi=C \eta=\left(B^{+} P_{W}+B^{-} P_{W \perp}\right) \eta .
$$

So applying $B^{+}$one has $\xi=B^{+} P_{W} \eta=B^{+} \tau$, where $\tau=P_{W} \eta \in W$. Hence the task at hand is to prove

Lemma 3.1. $C$ is bijective.

Proof. We show that $\operatorname{Ker}(C)=0$ and ind $(C)=0$. So suppose first that $0=C \psi=$ $B^{+} P_{W} \psi+B^{-} P_{W \perp} \psi$. Since $B^{+}$and $B^{-}$are orthogonal projections it follows that $B^{+} P_{W} \psi=0$ and $B^{-} P_{W \perp} \psi=0$. Then because $\beta=0$, and because $P_{W}+P_{W \perp}=I$ and $P_{W} \psi \in W$,

$$
0=\int_{Y}\left(B^{-} P_{W} \psi, P_{W} \psi\right) d y=\int_{Y}\left\|B^{-} P_{W} \psi\right\|^{2} d y
$$

and so $B^{-} P_{W} \psi=0$, and hence $P_{W} \psi=0$. Similarly, one has $P_{W \perp} \psi=0$, and so $\psi=0$ and that proves $\operatorname{Ker}(C)=0$.

To see that ind $(C)=0$ let $\zeta \in T_{y}^{*} Y$ with norm one and write the leading symbol $\sigma\left(D_{Y}\right)(y, \zeta)$ of $D_{Y}$ as $\sigma_{Y}(y, \zeta)$. Then from Lemma (2.1) and since

$$
\sigma(y, d u)^{2}=\sigma_{Y}(y, \zeta)^{2}=-1
$$

we have

$$
\sigma\left(P^{ \pm}\right)(y, \zeta)=1 / 2\left(I \mp i \sigma(y, d u) \sigma_{Y}(y, \zeta)\right) .
$$

Because $W \in G r$ and $W^{\perp} \in G r^{-}$, then

$$
\sigma\left(P_{W}\right)(y, \zeta)=\sigma\left(P^{+}\right)(y, \zeta) \text { and } \sigma\left(P_{W \perp}\right)(y, \zeta)=\sigma\left(P^{-}\right)(y, \zeta) .
$$

However, $\sigma\left(B^{ \pm}\right)(y, \zeta)=B^{ \pm}(y)=1 / 2\left(I \mp i \sigma_{Y}(y, \zeta)\right)$, and so one calculates

$$
\sigma(C)(y, \zeta)=\frac{1}{2}\left(I+\sigma_{Y}(y, \zeta)\right)
$$

Therefore $\sigma\left(C^{2}\right)(y, \zeta)=\frac{1}{2} \sigma(y, \zeta)$ is skew-adjoint and so ind $\left(C^{2}\right)=0$. But $\operatorname{ind}\left(C^{2}\right)=$ 2 ind $(C)$ and so ind $(C)=0$. That proves the lemma and hence that $B^{+}: W \rightarrow F^{+}$ is surjective. 
By assumption, $\beta(\phi, \phi ; D)=0$ for all $\phi \in W$ and so

$$
0=\int_{Y}\left(B^{+} \phi-B^{-} \phi, \phi\right) d y=\int_{Y}\left\|B^{+} \phi\right\|^{2}-\left\|B^{-} \phi\right\|^{2} d y,
$$

which proves that $g$ is well-defined, unitary and injective.

To show that $g$ is surjective we must prove that if $\eta \in L^{2}(Y ; S)$ is orthogonal to the range of $g$ then $\eta=0$. In fact, since it is clear from the definition of $g$ that it has invertible symbol on the unit sphere bundle and hence that it is elliptic, then we know $\eta \in C^{\infty}(Y ; S)$. Because $B^{+} \eta=0$ and $B^{+}$is self-adjoint, and because $B^{-} P_{W} \eta=g\left(B^{+} P_{W} \eta\right) \in \operatorname{Im}(g)$, then (12) implies

$$
\|\eta\|^{2}=\left\langle\left(B^{+}+B^{-}\right)\left(P_{W}+P_{W \perp}\right) \eta, \eta\right\rangle=\left\langle B^{-} P_{W \perp} \eta-B^{+} P_{W \perp} \eta, P_{W \perp} \eta\right\rangle,
$$

where we use the equality $\left\|P_{W} \eta\right\|^{2}=\left\langle P_{W} \eta, \eta\right\rangle=\left\langle B^{-} P_{W} \eta, \eta\right\rangle=0$. Hence, because if $W$ is maximal isotropic for the form $\beta$ then so is $W^{\perp}$, we have

$$
\|\eta\|^{2}=\left\langle i \sigma P_{W \perp} \eta, P_{W \perp} \eta\right\rangle=0 .
$$

It remains only to show that $g$ differs from $g_{+}$by a smoothing operator. However, that is clear, for

$$
g_{W}-g_{+}=B^{-}\left(P_{W}-P^{+}\right) C^{-1} i_{+}=\frac{1}{2} B^{-}\left(J_{W}-J\right) C^{-1} i_{+},
$$

and, because $W \in G r$, then $J_{W}-J$ is smoothing and the operators $B^{-}$and $C^{-1}$ are pseudodifferential operators of order 0 .

\section{Determinant Lines}

In this section we define the various determinant line bundles associated to the family of Dirac boundary value problems parameterized by the restricted Grassmannian. Using Theorem (1.1) we hence see the underlying topological reasons for the existence of the identifications in Theorems (1.2)-(1.5).

We begin by first recalling the construction of the determinant line bundle from [18]. This depends on the fact that an exact sequence of finite-dimensional vector spaces

$$
0 \longrightarrow V_{0} \longrightarrow V_{1} \stackrel{a}{\longrightarrow} V_{2} \longrightarrow V_{3} \longrightarrow 0
$$

(with $\operatorname{dim} V_{1}=\operatorname{dim} V_{2}$ ) defines a canonical isomorphism of complex lines

$$
\operatorname{Det} V_{1}^{*} \otimes \operatorname{Det} V_{2} \cong \operatorname{Det} V_{0}^{*} \otimes \operatorname{Det} V_{3} \text {. }
$$

Here Det $V$ denotes the top exterior power of $V$. The determinant $\operatorname{det} a \in \operatorname{Det} V_{1}^{*} \otimes$ Det $V_{2}$ of $a$ can therefore naturally be identified as an element of Det $V_{0}^{*} \otimes \operatorname{Det} V_{3}$. Consequently, if we consider an operator $a$ acting between infinite-dimensional vector spaces $V_{1}, V_{2}$ and if $a$ is Fredholm, then formally we may still make sense of $\operatorname{det} a$ as an element of the complex line

$$
L(a)=\operatorname{Det}(\operatorname{Ker} a)^{*} \otimes \operatorname{Det} \operatorname{Coker} a .
$$


Thus, in particular, the determinant map $W \mapsto \operatorname{det} D_{W}$ for the family of Dirac boundary value problems $\left\{D_{W}: W \in G r\right\}$ parameterized by the restricted Grassmannian arises as a canonical section det $: G r \rightarrow L$ of the holomorphic line bundle $L$ over $G r$ with fibre $L_{W}=L\left(D_{W}\right)$. More precisely, det is the section that picks out the element of $L_{W}$ which is mapped to 1 by the canonical isomorphism $L_{W} \cong C$ when $D_{W}$ has index zero and is invertible, and which otherwise is equal to zero.

$L$ is called the Quillen determinant line bundle of this family. ( $G r$ defines a holomorphic family of elliptic boundary value problems in the sense explained in Appendix A.) The bundle structure on $L$ is defined relative to the covering of $G r$ by open subsets $U_{y}$, with $y \in R^{+}$, parameterising those boundary value problems $D_{W}$ for which $y$ is not in the spectrum of the Laplacian $D_{W}^{*} D_{W}$, or $D_{W} D_{W}^{*}$. Over each $U_{y}$ are smooth finite-rank vector bundles $H_{y}^{+}, H_{y}^{-}$constructed as the sum of eigenspaces of the Laplacians for eigenvalues less than $y$, and from (15), (16) one obtains a canonical isomorphism

$$
L_{\mid U_{y}} \rightarrow \operatorname{Det}\left(H_{y}^{+}\right)^{*} \otimes \operatorname{Det} H_{y}^{-},
$$

which defines the determinant line bundle as a holomorphic vector bundle.

A metric $\|\cdot\|_{\zeta}$ is defined on $L$ by multiplying the induced metric from the $L^{2}$ metrics on $H_{y}^{ \pm}$by the regularised $\zeta$-function determinant $\operatorname{det}_{\zeta} D_{W}^{*} D_{W}$. One defines $\operatorname{det}_{\zeta} D_{W}^{*} D_{W}=\exp \zeta^{\prime}(0)$ when $D_{W}$ is invertible and 0 otherwise, where the zetafunction $\zeta(s)$ is defined for $\operatorname{Re}(s)>>0$ by $\zeta(s)=\operatorname{Tr}\left(D_{W}^{*} D_{W}\right)^{-s}$, and is defined around 0 by analytic continuation. In particular, $\left\|\operatorname{det} D_{W}\right\|_{\zeta}^{2}=\operatorname{det}_{\zeta} D_{W}^{*} D_{W}$. As with closed manifolds, the analytic continuation of the $\zeta$-function for the elliptic boundary conditions we are using follows from the existence of the asymptotic expansion of the heat kernel of the Laplacian. For an analysis of the heat kernel asymptotics for Atiyah-Patodi-Singer boundary conditions we refer to $[3,10,13,14]$. Heat kernel asymptotics for the whole Grassmannian of self-adjoint boundary conditions have been studied in [30].

There is, however, an alternative construction of the determinant line bundle due to Segal [25] which is more sensitive to the boundary condition. We recall his definitions. Let $V_{1}$ and $V_{2}$ be Frechet vector spaces and let $a: V_{1} \rightarrow V_{2}$ be a Fredholm operator of index zero. Then the (Segal) determinant line of $a$ is the complex line whose points are equivalence classes $[A, \lambda]$ of pairs $(A, \lambda)$, where $\lambda \in C$ and $A-a$ is trace-class. For $q=1+$ traceclass $: V_{1} \rightarrow V_{2}$ with $\operatorname{det} q \neq 0$ the equivalence relation is $(A q, \lambda) \sim(A, \lambda \operatorname{det} q)$. The line has a distinguished element $\operatorname{det} a=[a, 1]$ defined to be the determinant of $a$. If $a$ has index $n$ one defines Det $a=\operatorname{Det} a \oplus 0$, where $a \oplus 0: V_{1} \rightarrow V_{2} \oplus C^{n}$ if $n>0$, and $a \oplus 0: V_{1} \oplus C^{-n} \rightarrow V_{2}$ if $n<0$. Notice that Det $a$ is invariant under perturbation by a trace-class operator $t: V_{1} \rightarrow V_{2}$, that is, $\operatorname{Det}(a+t)=\operatorname{Det} a$.

The following properties [25] of the determinant line Det $a$ are fundamental.

\section{Proposition 4.1 [25]}

1. If $a: V_{1} \rightarrow V_{2}$ is a Fredholm operator there is a canonical isomorphism of determinant lines

Det $a \cong L(a)$. 
2. Let

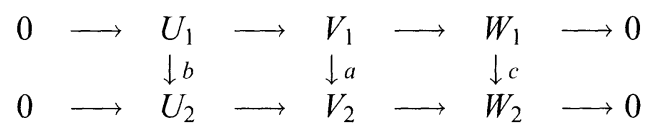

be a commutative diagram of topological vector spaces with exact rows and Fredholm columns. Then there is a canonical isomorphism

$$
\text { Det } a \cong \operatorname{Det} b \otimes \operatorname{Det} c \text {, }
$$

depending holomorphically on $a, b, c$. If $a, b, c$ are invertible it preserves the determinant elements:

$$
\operatorname{det} a \leftrightarrow \operatorname{det} b \otimes \operatorname{det} c .
$$

Proof. It is enough to prove (18) for the case ind $a=0$. Let $e_{1}, \ldots, e_{r}$ be a basis for $\operatorname{Ker}(a)$ regarded as being in $V_{0}$, and let $f_{1}, \ldots, f_{r}$ be a basis for $\operatorname{Coker}(a)$. The isomorphism (18) is defined by

$$
\left[a+\sum_{l=1}^{r} f_{l} \otimes e_{i}^{*}, 1\right] \leftrightarrow e_{1}^{*} \wedge \ldots \wedge e_{r}^{*} \otimes f_{1} \wedge \ldots \wedge f_{r} .
$$

If $a, b, c$ are invertible operators the isomorphism (19) is defined by (20). If not, then the operators are modified to invertible operators by adding to each a suitable finite rank operator, chosen so as to preserve the commutativity of the diagram, and then mapping between determinant elements.

The Segal determinant line bundle of the family of Dirac boundary value problems parameterized by $G r$ is the holomorphic line bundle $\mathscr{L}$ over $G r$ whose fibres are

$$
\mathscr{L}_{W}=\operatorname{Det} D_{W} .
$$

The bundle structure of $\mathscr{L}$ follows from the general constructions given in [25]. More precisely, over $G r \times X$ one has a Hermitian bundle of spinors $\mathscr{S}$ which restricts over each fibre to the spinor bundle $S$ over $X$. It is enough to work with open sets of $G r$, where the bundle $\pi_{*}(\mathscr{S})$ over $G r$, whose fibre at $W \in G r$ is the space of sections $C_{W}^{\infty}(X ; S)$, is trivial and ind $D_{W}=0$. Specifically we use the covering of $G r$ by open sets $U_{b}$, where $b: C^{\infty}(X ; S) \rightarrow C^{\infty}(X ; S)$ is a finite rank operator, consisting of points $W$ of $G r$ for which $D_{W}+b$ is invertible. A specific trivialization over $U_{b}$ is defined by the gauge

$$
U_{b} \longrightarrow \mathscr{L}_{\mid U_{b}}, \quad W \longmapsto \operatorname{det} D_{W}+b .
$$

Patching together the locally defined complex line bundles over each intersection $U_{b} \cap U_{c}$ by the transition function

$$
W \mapsto \operatorname{det}\left(D_{W}+c\right)\left(D_{W}+b\right)^{-1}=\operatorname{det}\left(1+(c-b)\left(D_{W}+b\right)^{-1}\right),
$$

which depends holomorphically on $W$, defines the determinant line bundle globally. $\mathscr{L}$, like the Quillen determinant line bundle $L$, has a canonical determinant section, which we also denote by det, over the index zero component of $\mathrm{Gr}$ defined by $\operatorname{det} D_{W}=\left[D_{W}, 1\right]$ if $D_{W}$ is invertible, and zero otherwise.

Proposition 4.2. There is a canonical isomorphism of determinant line bundles

$$
\mathscr{L} \cong L
$$

which preserves the determinant sections. 
Proof. This is an immediate consequence of Proposition (4.1)(1). That the determinant elements are preserved follows from the definition of the isomorphism (21).

There are, however, many more determinant line bundles over $G r$ arising from the fact that for any two points $W_{0}, W_{1} \in G r$ there is a canonical Fredholm operator

$$
\rho_{W_{0}}\left(W_{1}\right)=P_{W_{1}} \circ i_{W_{0}}: W_{0} \rightarrow W_{1}
$$

defined by inclusion followed by orthogonal projection. Following [25] one can define the determinant line $\operatorname{Det}\left(W_{0}: W_{1}\right)$ to be $\operatorname{Det} \rho_{W_{0}}\left(W_{1}\right)$, and there is an obvious canonical isomorphism

$$
\operatorname{Det}\left(W_{0}: W_{1}\right) \otimes \operatorname{Det}\left(W_{1}: W_{2}\right) \cong \operatorname{Det}\left(W_{0}: W_{2}\right) \text {. }
$$

For each $W_{0} \in G r$ the operators $\rho_{W_{0}}(W)$ depend holomorphically on $W$ and hence one has a holomorphic line bundle based at $W_{0}$,

$$
\operatorname{Det}_{W_{0}}=\bigcup_{W \in G r} \operatorname{Det}\left(W_{0}: W\right)
$$

where the bundle structure is defined precisely as for $D_{W}$ (with $D_{W}$ replaced by $\rho_{W_{0}}(W)$ ), From (22) we obtain for any $W_{0}, W_{1} \in G r$ a canonical isomorphism

$$
\operatorname{Det}_{W_{1}} \cong \operatorname{Det}_{W_{0}} \otimes \operatorname{Det}\left(W_{0}: W_{1}\right)
$$

In particular, Det $_{H^{+}}$is the determinant line bundle of [17].

We identify the determinant line bundle of the family of Dirac boundary value problems $\left\{D_{W}: W \in G r\right\}$ as follows.

Proposition 4.3. There is a canonical isomorphism of holomorphic line bundles

$$
\mathscr{L} \cong \operatorname{Det}_{K}
$$

When $D_{W}$ is invertible $\operatorname{det} D_{W}$ maps to $\operatorname{det} \rho_{K}(W)$.

Proof. We must exhibit a canonical isomorphism of determinant lines

$$
\operatorname{Det} D_{W} \cong \operatorname{Det}(K: W)=\operatorname{Det} \rho_{K}(W)
$$

that depends holomorphically on $W \in G r$. To do that, note the operator

$$
(D ; W): C^{\infty}(X ; S) \rightarrow C^{\infty}(X ; S) \oplus W, \quad \psi \rightarrow\left(D \psi, P_{W} b \psi\right),
$$

fits into the following commutative diagram with exact rows:

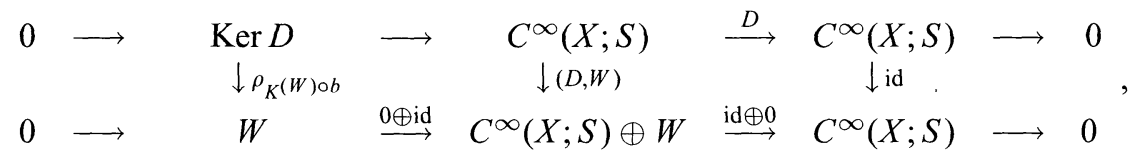

and, since $W \in G r$, the vertical maps are all Fredholm. Hence Proposition 4.1 identifies a canonical isomorphism

$$
\operatorname{Det}(D ; W) \cong \operatorname{Det}\left(\rho_{K}(W) \circ b\right) \otimes \operatorname{Det} i d=\operatorname{Det}\left(\rho_{K}(W) \circ b\right)=\operatorname{Det} \rho_{K}(W) .
$$


The final equality follows from Lemma (2.3). There is also the commutative diagram with exact rows

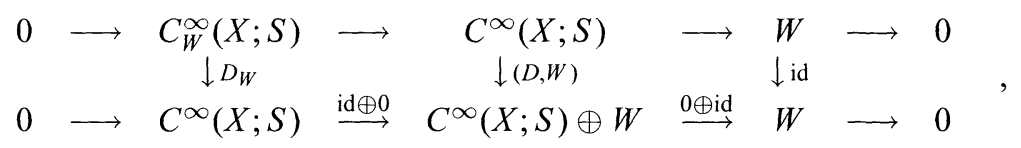

and hence a canonical isomorphism

$\operatorname{Det}(D ; W) \cong \operatorname{Det} D_{W} \otimes \operatorname{Det} i d=\operatorname{Det} D_{W}$.

By Proposition 4.1 the isomorphisms vary holomorphically with $W$ and preserve the determinant elements when $D_{W}$ is invertible. That completes the proof.

That the same statement also holds for the Quillen determinant line bundle is an immediate consequence of Proposition (4.2), however one may also deduce it directly from the following commutative diagram with exact columns and rows:

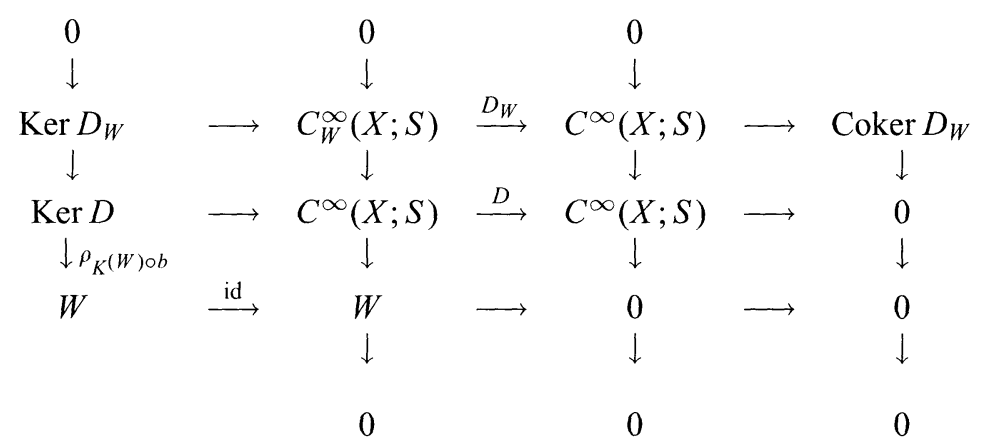

The exactness of the second row is a consequence of the following lemma.

Lemma 4.1. $D: C^{\infty}(X ; S) \rightarrow C^{\infty}(X ; S)$ is surjective.

Proof. Let $M=X \cup_{Y} X^{-}$be the closed double manifold. Since the restriction map $r: C^{\infty}\left(M ; S_{M}\right) \rightarrow C^{\infty}(X ; S)$ is surjective, it is enough to show that

$$
\operatorname{range}\left(D_{M}\right)+\operatorname{Ker}(r)=C^{\infty}\left(M ; S_{M}\right),
$$

for then given $\psi \in C^{\infty}(X ; S)$ there is a $\xi \in \operatorname{range}\left(D_{M}\right)$ with

$$
\psi=r(\xi)=r D_{M}(\tau)=\operatorname{Dr}(\tau),
$$

for some $\tau \in C^{\infty}\left(M ; S_{M}\right)$. The required identity holds because if $t$ is a linear form on $C^{\infty}\left(M ; S_{M}\right)$ which vanishes on the left-hand side of the identity, then $t$ is a distributional solution of $D_{M}^{*} t=0$ with support in $X^{-}$. By elliptic theory on closed manifolds $t$ is smooth and hence by the unique continuation property $t=0$ over all of $M$.

Now using the Snake Lemma of [15](p. 202) and Lemma (2.3) we obtain from the outside columns of the diagram an exact sequence

$$
0 \longrightarrow \operatorname{Ker} D_{W} \longrightarrow K \stackrel{\rho_{K}(W)}{\longrightarrow} W \longrightarrow \operatorname{Coker} D_{W} \longrightarrow 0
$$


and hence canonical isomorphisms $\operatorname{Ker} \rho_{K}(W) \cong \operatorname{Ker} D_{W}$ and $\operatorname{Coker} \rho_{K}(W) \cong$ Coker $D_{W}$, which define fibrewise the asserted isomorphism of determinant line bundles.

Notice that it is the exact sequence in the top row of the diagram that identifies $\operatorname{det} D_{W}$ as an element of the complex line $L_{W}$ in precisely the same way as for elliptic operators over closed manifolds. The diagram extends this exact sequence because of the additional dependence on the boundary condition. We also deduce the important fact that the determinant line bundle and hence $\operatorname{det} D_{W}$ are completely determined by the boundary data.

If we specialize to the case where $X$ is 1-dimensional and connected, and $D=i \nabla$, where $\nabla$ is a Hermitian connection on a complex $n$-bundle $\mathscr{E}$ over $X$, then the restricted Grassmannian is just the usual finite-dimensional complex Grassmannian $G_{2 n}$. In particular, $W$ and $K$ are finite-dimensional and $K$ is the graph of the parallel transport of the connection $\nabla$. We recall that $G r_{2 n}$ is a compact Kähler manifold consisting of connected components $G r_{k, 2 n}$ parameterizing $k$-dimensional subspaces of $\mathscr{E}_{0} \oplus \mathscr{E}_{1} \cong C^{2 n}$, where $\mathscr{E}_{0}, \mathscr{E}_{1}$ are the boundary fibres, and the isomorphism depends on a choice of frame. We have the following specific identification of the determinant line bundle.

Proposition 4.4. Let $\operatorname{dim} X=1$. There is a canonical isomorphism of holomorphic line bundles over $G r_{k, 2 n}$,

$$
L \cong \operatorname{Det} \xi \otimes \operatorname{Det} \mathscr{K}^{*},
$$

where $\xi$ is the tautological bundle of rank $k$ with fibre at a point of $G r_{k, 2 n}$ equal to the corresponding subspace of $C^{2 n}$, and $\mathscr{K}$ denotes the trivial bundle with fibre $K$. Any other holomorphic line bundle over $G r_{k, 2 n}$ is isomorphic to $\mathscr{L}^{\otimes p}$ for some integer $p$.

Proof. The only extra piece of data we need to prove this is that the first Chern class defines an isomorphism of sheaf cohomology groups $c_{1}: H^{1}\left(G r_{k, 2 n}, \vartheta^{*}\right) \longrightarrow$ $H^{2}\left(G r_{k, 2 n} ; Z\right.$ ). Here $\vartheta$ (resp. $\left.\vartheta^{*}\right)$ denotes the sheaf of holomorphic (resp. nonzero holomorphic) functions on $G r_{k, 2 n}$. We recall for the convenience of the reader why that is true. Isomorphism classes of holomorphic line bundles over $G r_{k, 2 n}$ are parameterised by the sheaf cohomology group $H^{1}\left(G r_{k, 2 n} ; \vartheta^{*}\right)$ which fits into the exact sequence

$$
0 \rightarrow H^{1}\left(G r_{k, 2 n}, \vartheta\right) \rightarrow H^{1}\left(G r_{k, 2 n}, \vartheta^{*}\right) \rightarrow H^{2}\left(G r_{k, 2 n}, Z\right) \rightarrow H^{2}\left(G r_{k, 2 n}, \vartheta\right)
$$

induced from the short exact sequence $0 \rightarrow Z \rightarrow \vartheta \stackrel{\exp }{\rightarrow} \vartheta^{*} \rightarrow 0$. The cohomology ring $H^{*}\left(G r_{k, 2 n}\right)$ is a polynomial ring generated by the Chern classes $c_{1}(\xi), \ldots, c_{k}(\xi)$ subject to the condition on the total Chern class $c(\xi) c\left(\xi^{\perp}\right)=1$. Hence over a ground ring $R$ one has

$$
H^{1}\left(G r_{k, 2 n} ; R\right)=0 \quad \text { and } \quad H^{2}\left(G r_{k, 2 n} ; R\right)=R .
$$

There is a Hodge decomposition $H^{1}\left(G r_{k, 2 n}\right)=H^{1,0}\left(G r_{k, 2 n}\right) \oplus H^{0,1}\left(G r_{k, 2 n}\right)$ because $G r_{k, 2 n}$ is Kähler which combined with (25) and the Dolbealt isomorphism gives $H^{1}\left(G r_{k, 2 n} ; \vartheta\right)=0$. Also, from (26) and Hodge decomposition, one has

$$
C=H^{2}\left(G r_{k, 2 n}\right) \cong H^{2,0}\left(G r_{k, 2 n}\right) \oplus H^{1,1}\left(G r_{k, 2 n}\right) \oplus H^{0,2}\left(G r_{k, 2 n}\right),
$$

and since $H^{1,1}\left(G r_{k, 2 n}\right)=C[\omega]$, where $\omega$ is the Kähler form on $G r_{k, 2 n}$, it follows that $H^{2}\left(G r_{k, 2 n}, \vartheta\right) \cong H^{0,2}\left(G r_{k, 2 n}\right)=0$. The exact sequence $(25)$ thus reduces to the asserted isomorphism of Abelian groups. 
In particular, the determinant line bundle is a holomorphic line bundle over $G r_{k, 2 n}$ and hence is classified by its first Chern class. Since $W$ and $K$ are finitedimensional one has from the exact sequence (24) a canonical isomorphism

$$
L_{W} \cong \operatorname{Det} K^{*} \otimes \operatorname{Det} W \text {, }
$$

which defines fibrewise the required line bundle isomorphism. That implies $c_{1}(\mathscr{L})=$ $c_{1}$ (Det $\xi$ ) and because from $(26) H^{2}\left(G r_{k, 2 n} ; Z\right)=Z$ and $c_{1}$ (Det $\left.\xi\right)=c_{1}(\xi)=-1$ we see every holomorphic line bundle over $G r_{k, 2 n}$ is isomorphic to $\mathscr{L}^{\otimes p}$ for some integer $p$.

In fact, because all the boundary conditions are finite-dimensional when $\operatorname{dim} X=$ 1 one has more generally

$$
\operatorname{Det}_{W_{0}} \cong \operatorname{Det} \xi \otimes \operatorname{Det} \mathscr{W}_{0}^{*},
$$

where $\mathscr{W}_{0}$ is the trivial bundle with fibre $W_{0}$, and the isomorphism (23) is just the identification $\operatorname{Det}\left(W_{0}: W_{1}\right)=\operatorname{Det} W_{0}^{*} \otimes \operatorname{Det} W_{1}$.

Returning to the general case we have

Proposition 4.5. Let $X$ be an odd-dimensional spin manifold. Then the determinant line bundle is canonically trivial over $G r_{\text {iso. }}$.

Proof. From Proposition (4.3) the line bundle $\mathscr{L}$ has fibre $\mathscr{L}_{W} \cong \operatorname{Det}(K: W)$ at

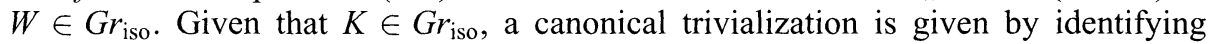
$W$ and $K$ with $F^{+}$by the projection maps $W \subset F \rightarrow F^{+}$and $K \subset F \rightarrow F^{+}$defined by Theorem (1.1).

It remains to prove

Lemma 4.2. $K \in G r_{\text {Iso. }}$.

Proof. We know from Proposition (2.2) that $K \in G r$, and so by Theorem (1.1) it is enough to show that $K=\Gamma(h)$ for some unitary $h: F^{+} \rightarrow F^{-}$differing from $g_{+}$ by a smoothing operator. Green's formula states that for $\psi_{0}, \psi_{1} \in C^{\infty}(X ; S)$,

$$
\int_{X}\left(\left\langle D \psi_{0}, \psi_{1}\right\rangle-\left\langle\psi_{0}, D \psi_{1}\right\rangle\right) d x=\int_{Y} i\left\langle B^{+} b \psi_{0}-B^{-} b \psi_{1}, b \psi_{1}\right\rangle d y,
$$

where $B^{ \pm}$are as in Sect. 3. Thus if $D$ is endowed with the boundary condition $W=K^{\perp}$, the left-hand side vanishes (by Lemma (2.3)). Repeating the arguments in the proof of Theorem (1.1) identifies an $L^{2}$ unitary isomorphism $u: F^{-} \rightarrow F^{+}$ of the form

$$
u=i D^{-}\left(D^{+} D^{-}\right)^{-1 / 2}+s,
$$

where $s: F^{-} \rightarrow F^{+}$is smoothing. Because $K^{\perp}$ is in the opposite Grassmannian $\mathrm{Gr}^{-}$ the roles of $F^{+}$and $F^{-}$are reversed. Because $K^{\perp}=\Gamma(u)$ implies $K=\Gamma\left(-u^{*}\right)$, taking $h=-u^{*}$ completes the proof.

We note that the triviality (though not a preferred trivialization) of the determinant line bundle over $G r_{\text {iso }}$ also follows from the computation of the homotopy groups of the Grassmannian made in [7 and 10] (Appendix B). That $K$ defines a self-adjoint boundary condition for $D$ is known [7].

It is clear that the given trivialization of $\mathscr{L}_{\mid G r_{\text {iso }}}$ can be extended to an open neighbourhood of the real submanifold $G r_{\text {iso }}$ of the Grassmannian by including all boundary conditions $W$ which are the graphs of invertible maps $g: F^{+} \rightarrow F^{-}$ 
such that $g-g_{+}$is smoothing. For example, in the simplest case when $X=[0,2 \pi]$ with $D=i \frac{d}{d x}$ and $n=1$, the isotropic Grassmannian $G r_{1 s o, 2} \cong U(1)$, and hence self-adjoint boundary conditions for $D$, correspond to the equatorial circle on $C P^{1}$ (identified with $S^{2}$ ). The map $i: U(1) \rightarrow C P^{1}$ extends to a map $i: C^{*} \rightarrow C P^{1}$ from the non-zero complex numbers $C^{*}=G l(1 ; C)$, and the pull-back bundle $i^{*}(\mathscr{L})$ is trivial. That is, the holomorphic line bundle obtained from $\mathscr{L} \rightarrow C P^{1}$ by deleting points at 0 and $\infty$ can be trivialized and hence a holomorphic determinant function identified once a trivialization is chosen. Note that the operators $D_{0}$ and $D_{\infty}$ have no spectrum.

Proposition (4.5) is the underlying topological reason for the existence of the identifications in Theorem (1.2) and Theorem (1.3). Combined with the next theorem this also yields the topology behind Theorem (1.4) and Theorem (1.5). To state this theorem, let $X^{0}, X^{1}$ be Riemannian spin manifolds each with boundary (with reverse orientations) $Y$ and with Dirac operators $D^{0}, D^{1}$. Over the closed double manifold $M=X^{0} \cup_{Y} X^{1}$ one has the bundle $S_{M}$ and first-order elliptic operator $D_{M}: C^{\infty}\left(M ; S_{M}\right) \rightarrow C^{\infty}\left(M ; S_{M}\right)$, exactly as defined in Sect. 1. Let $\mathscr{L}^{0}, \mathscr{L}^{1}$ be the respective determinant line bundles of the families of Dirac boundary value problems $D_{W}^{0}, D_{W \perp}^{1}$, parameterized by the restricted Grassmannian $G r$ of elliptic boundary conditions for $D^{0}$. Let $\mathscr{L}_{M}=\operatorname{Det} D_{M} \times G r$ be the trivial line bundle over $G r$ with fibre the determinant line $\operatorname{Det} D_{M}$.

Theorem 4.1. There is a canonical isomorphism of determinant line bundles

$$
\mathscr{L}_{M} \cong \mathscr{L}^{0} \otimes \mathscr{L}^{1}
$$

When $D_{M}$ is invertible and with the harmonic spinor boundary condition $W=K^{0}$ the determinant elements are preserved, that is,

$$
\operatorname{det} D_{M} \leftrightarrow \operatorname{det} D_{K^{0}}^{0} \otimes D_{K^{1}}^{1} .
$$

So as not to detour too long from our aim of calculating determinants we have placed the proof of Theorem (4.1) in Appendix B. (The proof of this theorem with minor modifications holds for general smooth families of Dirac boundary value problems, in which case the left-hand side of (27) will not in general be a trivial bundle. This extends the corresponding result of Segal [25] for families of $\bar{\partial}$-operators over a Riemann surface with the Atiyah-Patodi-Singer boundary condition.)

We may restate Theorem (4.1) as follows.

Corollary 4.1. There is a canonical isomorphism of complex line bundles

$$
\mathscr{L}_{M} \cong \operatorname{Det}_{K^{0}} \otimes \operatorname{Det}_{K^{1}}
$$

Here $\operatorname{Det}_{K^{1}}$ refers to the line bundle with fibre $\operatorname{Det}\left(K^{1}: W^{\perp}\right)$ (rather than $\operatorname{Det}\left(K^{1}\right.$ : $W$ ). We may in fact deduce a little more. Not only is $\operatorname{Det}_{K^{0}} \otimes \operatorname{Det}_{K^{1}}$ trivial over all of $G r$ but, by Proposition (4.5), its restriction to $G r_{\text {iso }}$ has a preferred tirvialization, which we use in Sect. 5 to calculate the determinants. In dimension 1 we may see that explicitly as follows. Let $S^{1}=X^{0} \cup X^{1}$ and let $D_{S^{1}}, D^{0}, D^{1}$ be as in the statement of Theorem (1.5) (Sect. 1).

Corollary 4.2. There is a canonical isomorphism of trivial holomorphic line bundles over $G r_{k, 2 n}$,

$$
\mathscr{L}_{S^{1}} \cong \operatorname{Det}\left(\xi \oplus \xi^{\perp}\right) \otimes \operatorname{Det} \mathscr{K}^{0} \otimes \operatorname{Det} \mathscr{K}^{1}
$$


where $\mathscr{K}^{i}$ is the trivial bundle of rank $k$ with fibre $K^{i}$. Over the isotropic Grassmannian $\mathscr{L}_{S^{1}}$ is canonically isomorphic to the trivial line bundle $G r_{\text {iso, } 2 \mathrm{n}} \times C$.

Proof. With $W$ replaced by $W^{\perp}$ one has a canonical isomorphism $\mathscr{L}^{1} \cong \operatorname{Det}\left(\xi^{\perp}\right) \otimes$ Det $\mathscr{K}^{1}$ exactly as in Proposition (4.4). Hence, since $\operatorname{Det} \xi \otimes \operatorname{Det}\left(\xi^{\perp}\right) \cong \operatorname{Det}(\xi \oplus$ $\xi^{\perp}$ ) and $\xi \oplus \xi^{\perp}$ is trivial, then Theorem (4.1) implies the first statement. Over the isotropic Grassmannian each fibre of $\xi$ (resp. $\xi^{\perp}$ ) is the graph of some unitary isomorphism of the boundary fibres of $\mathscr{E}^{0}$ (resp. $\mathscr{E}^{1}$ ), and hence one has canonically $\xi \oplus \xi^{\perp} \cong G r_{\text {iso, } 2 \mathrm{n}} \times C^{2 n}$ by projection in each fibre. With the corresponding idendifications for $\mathscr{K}^{0}$ and $\mathscr{K}^{1}$, Theorem (4.1) proves the second statement.

\section{Proof of Theorems (1.2) and (1.3), and the Gauge Determinant}

5a. Proof of Theorem (1.2) and (1.3). Let $W \in G r$ and define

$$
\mathscr{C}_{W}: K \oplus W^{\perp} \rightarrow F, \quad \mathscr{C}_{W}(\phi, \varphi)=\frac{1}{\sqrt{2}}\left(i_{K}(\phi)+i_{W \perp}(\varphi)\right),
$$

where $i_{K}: K \rightarrow F$ and $i_{W \perp}: W^{\perp} \rightarrow F$ are the inclusion maps.

Proposition 5.1. $\mathscr{C}_{W}$ is Fredholm and there is a canonical isomorphism of determinant lines

$$
L_{W} \cong L\left(\mathscr{C}_{W}\right),
$$

which takes $\operatorname{det} D_{W}$ to $\operatorname{det} \mathscr{C}_{W}$ when $D_{W}$ is invertible.

Proof. We have the following commutative diagram with exact columns and rows:

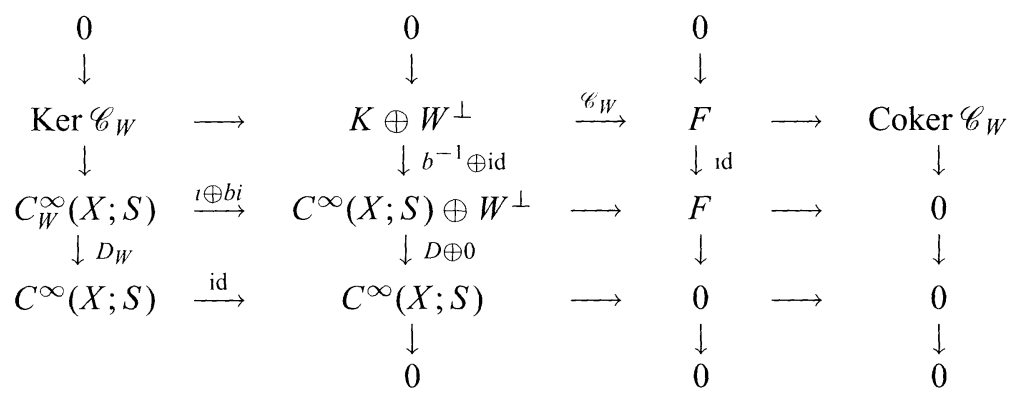

In the second row $i$ denotes the inclusion and the central map is $(\psi, \varphi) \rightarrow$ $\frac{1}{\sqrt{2}}\left(b \psi+i_{W \perp} \varphi\right)$. That $\operatorname{Ker} D_{W} \cong \operatorname{Ker} \mathscr{C}_{W}$ is clear since $b_{\mid \operatorname{Ker} D}$ is bijective. The exactness of the second column is because $D: C^{\infty}(X ; S) \rightarrow C^{\infty}(X ; S)$ is surjective (Lemma (4.1)).

The Snake Lemma [15] (p. 202) picks out from the diagram an exact sequence

$$
0 \longrightarrow \operatorname{Ker} \mathscr{C}_{W} \longrightarrow C_{W}^{\infty}(X ; S) \stackrel{D_{W}}{\longrightarrow} C^{\infty}(X ; S) \longrightarrow \text { Coker } \mathscr{C}_{W} \longrightarrow 0,
$$

and hence canonically identifies the determinant of $D_{W}$ as an element $\operatorname{det}_{\mathscr{C}} D_{W}$ of the complex line $\operatorname{Det}\left(\operatorname{Ker}^{C} \mathscr{C}_{W}\right)^{*} \otimes \operatorname{Det}\left(\operatorname{Coker} \mathscr{C}_{W}\right)=L\left(\mathscr{C}_{W}\right)$. The asserted isomorphism of determinant lines is thus defined by mapping between the generators

$$
\operatorname{det} D_{W} \leftrightarrow \operatorname{det}_{\mathscr{C}} D_{W},
$$


which identifies $\operatorname{det}_{\mathscr{C}} D_{W}$ as the unique element of $L\left(\mathscr{C}_{W}\right)$ which maps to 1 under the canonical isomorphism with $C$ when $\mathscr{C}_{W}$ is invertible. Hence, by definition of the determinant sections, $\operatorname{det}_{\mathscr{C}} D_{W}$ coincides with $\operatorname{det} \mathscr{C}_{W}$.

For $W \in G r_{\text {iso }}$ we now define the canonical determinant of the Dirac boundary value problem $D_{W}$ to be $\operatorname{det}_{\mathscr{C}} D_{W}=\operatorname{det} \mathscr{C}_{W}$ when $\operatorname{Ker} D_{W}=0$, and 0 otherwise.

To see why that is equal to the asserted complex number let $g: F^{+} \rightarrow F^{-}$and $h: F^{+} \rightarrow F^{-}$be the unitary isomorphisms defining $W$ and $K$ (by Lemma (4.2)).

From the top row of the diagram one now has an exact sequence

$$
0 \longrightarrow \operatorname{Ker} D_{W} \longrightarrow K \oplus W^{\perp} \longrightarrow F \longrightarrow \operatorname{Coker} D_{W} \longrightarrow 0,
$$

and when $D_{W}$ is invertible then $\mathscr{C}_{W}: K \oplus W^{\perp} \rightarrow F$ is an isomorphism, and by definition

$$
\mathscr{C}_{W}\left(\left(\phi^{+}, h \phi^{+}\right),\left(-g^{-1} \varphi^{-}, \varphi^{-}\right)\right)=\frac{1}{\sqrt{2}}\left(\phi^{+}-g^{-1} \varphi^{-}, h \phi^{+}+\varphi^{-}\right) .
$$

So, with respect to the polarization $F=F^{+} \oplus F^{-}$into positive and negative spinor fields, the canonical determinant is given by

$$
\operatorname{det}_{\mathscr{C}} D_{W}=\operatorname{det} \frac{1}{\sqrt{2}}\left(\begin{array}{cc}
1 & -g^{-1} \\
h & 1
\end{array}\right) .
$$

Hence because of the factorization into upper and lower triangular matrices

$$
\left(\begin{array}{cc}
1 & g_{0} \\
h & 1
\end{array}\right)=\left(\begin{array}{cc}
1 & g_{0} \\
0 & 1
\end{array}\right)\left(\begin{array}{cc}
1-g_{0} h & 0 \\
h & 1
\end{array}\right),
$$

where $g_{0}=-g^{-1}$, and the identity $\operatorname{det}(1+a) \operatorname{det}(1+b)=\operatorname{det}(1+a+b+a b)$ for trace-class operators $a, b$ there is the canonical identification

$$
\operatorname{det}_{\mathscr{C}} D_{W}=\operatorname{det} \frac{1}{2}\left(1-g_{0} h\right)
$$

which by definition [27] exists as a number in $C$ because $\frac{1}{2}\left(1-g_{0} h\right)$ is of the form $1+$ smoothing. That completes the proof of Theorem (1.2).

5b. Proof of Theorem (1.3). Let $X$ be a compact connected 1-manifold with boundary. We consider the first-order elliptic operator $D=i \nabla_{d / d x}: C^{\infty}(X ; \mathscr{E}) \rightarrow$ $C^{\infty}(X ; \mathscr{E})$. Then self-adjoint boundary conditions for $D$ are parameterised by the finite-dimensional isotropic Grassmannian $G r_{\text {iso, } 2 n}\left(\mathscr{E}_{0} \oplus \mathscr{E}_{1}\right)$ which is identified with the space of unitary isomorphisms $\mathscr{E}_{0} \rightarrow \mathscr{E}_{1}$. We assume a trivialization of $\mathscr{E}$ such that the Hermitian structure in each boundary fibre is the pull-back of the standard Hermitian metric on $C^{n}$. The isotropic Grassmannian is thus identified with $G r_{\text {iso }, 2 n}=G r_{\text {iso, } 2 n}\left(C^{2 n}\right)$, and hence with the unitary group $U(n)$. Relative to the trivialization we have $D=i \frac{d}{d x}+A(x)$, where $A(x): C^{n} \rightarrow C^{n}$ is a Hermitian matrix. The $\zeta$-function trivialisation of the determinant line $L_{W}$ is the trivialisation associated with the norm $\|\cdot\|_{\zeta}$. We define an explicit $\zeta$-function determinant $\mathscr{L} \rightarrow C$ with $\operatorname{det} D_{W} \mapsto \operatorname{det}_{\zeta} D_{W}$, and $\left|\operatorname{det}_{\zeta} D_{W}\right|=\left\|\operatorname{det} D_{W}\right\|_{\zeta}$. To define the $\zeta$-function for the non-positive operator $D_{W}=\left(i \frac{d}{d x}+A(x)\right)_{W}$ we follow the constructions of [22]. We shall take $X=[0,1]$. 
Formally one defines the $\zeta$-function of $D_{W}$ by

$$
\zeta_{D_{W}}(s)=\operatorname{Tr} D_{W}^{-s} .
$$

We make sense of this equation as follows. Let $\gamma$ denote a contour in $C$ consisting of a ray in the sector of $C$ with $\operatorname{Re} z<0, \operatorname{Im} z<0$ going from $-i \infty$ to a small circle of radius $\delta$ about the origin, traversing the circle clockwise into the sector of $C$ with $\operatorname{Re} z>0, \operatorname{Im} z<0$, and then returning along a ray to $-i \infty$. For $\lambda$ disjoint from the spectrum of $D_{W}$ the resolvent $\left(D_{W}-\lambda\right)^{-1}$ is a holomorphic function of $\lambda$ and along the rays one has the $L^{2}$ estimate $\left\|\left(D_{W}-\lambda\right)^{-1}\right\| \leqq|\operatorname{im}(\lambda)|^{-1}$. Hence for $\operatorname{Re}(s)>1$ we can define

$$
D_{W}^{-s}=\frac{1}{2 \pi i} \int_{\gamma} \lambda^{-s}\left(D_{W}-\lambda\right)^{-1} d \lambda
$$

where $\gamma$ does not enclose any poles of $\left(D_{W}-\lambda\right)^{-1}$ and the equation is taking place in the Banach space of bounded operators $L^{2}\left(X ; C^{n}\right) \rightarrow L^{2}\left(X ; C^{n}\right)$ with the operator norm.

Proposition 5.2. $\zeta_{D_{W}}(s)$ is well-defined and holomorphic for $\operatorname{Re}(s)>1$. For $W \in$ $G r_{\text {iso,2n }}$ it has a holomorphic extension to all of $C$.

Proof. Let $K_{\lambda}(x, y ; W)$ denote the Schwartz kernel of

$$
i\left(D_{W}-\lambda\right)^{-1}=\left(\frac{d}{d x}+i(\lambda-A(x))\right)^{-1}
$$

relative to the boundary condition $W$, so for any $v \in C^{n}$ the function $x \longmapsto$ $K_{\lambda}(x, y ; W) v$ satisfies $W$. Then $D_{W}^{-s}$ is an integral operator with kernel

$$
k_{s}(x, y ; W)=\frac{1}{2 \pi i} \int_{\gamma} \lambda^{-s} i K_{\lambda}(x, y ; W) d \lambda .
$$

Let $\tau_{\lambda}: X \rightarrow G l(n ; C)$ be the monodromy of the covariant derivative $i\left(D_{W}-\lambda\right)$ with respect to the initial condition $\tau_{0}(0)$; that is, $\tau_{\lambda}^{\prime}=-i(\lambda-A(x)) \tau_{\lambda}$ and $\tau_{\lambda}(x)=$ $e^{-l i x} \tau_{0}(x)$. Then it is straightforward to verify that depending on $\tau_{\lambda}$ and the choice of boundary condition $W$ there are $n \times n$ matrices $P_{i}, Q_{i}$ independent of $x, y$ and satisfying $P_{\lambda}-Q_{\lambda}=1$, such that

$$
K_{\lambda}(x, y ; W)= \begin{cases}\tau_{\lambda}(x) P_{\lambda} \tau_{\lambda}(y)^{-1} & \text { for } x<y \\ \tau_{\lambda}(x) Q_{\lambda} \tau_{\lambda}(y)^{-1} & \text { for } x>y .\end{cases}
$$

So $K_{\lambda}(x, y ; W)$ is an infinitely smooth function of $x, y$ off the diagonal and has a simple jump discontinuity when $x=y$. Moreover, if $\operatorname{Re}(s)>1=\operatorname{dim} X$, then $D_{W}^{-s}$ is a continuous function on $X \times X$. For although the jump in $K_{\lambda}$ (i.e. $K_{\lambda}(x, x+\varepsilon)-$ $\left.K_{\lambda}(x, x-\varepsilon)\right)$ is $i$, the jump in $k_{s}(x, y ; W)$ is $\frac{l}{2 \pi} \int_{\gamma} \lambda^{-s} d \lambda=0$, and hence $k_{s}(x, y ; W)$ is a continuous function of $x, y \in X$. Thus $D_{W}^{-s}$ is trace-class for $\operatorname{Re} s>1$ and

$$
\zeta_{D_{W}}(s)=\operatorname{Tr}_{L^{2}}\left(D_{W}^{-s}\right)=\int_{X} \operatorname{Tr}_{C}\left(k_{s}(x, x ; W)\right) d x
$$

is holomorphic. 
To see that $\zeta_{D_{W}}(s)$ has an analytic continuation to all of $C$ when $W \in G r_{\text {iso,2n }}$ we must see that $K_{\lambda}(x, y ; W)$ has the right convergence behaviour as $\lambda \rightarrow-i \infty$. First, we choose $\tau_{\lambda}$ so that $\tau_{\lambda}(0)=1$. Then $\tau_{\lambda}(1)=h_{\lambda}=e^{-i \lambda} h$ is the monodromy of the covariant derivative $i(\lambda-D)$, where $h=h_{0}$, and clearly $K=\Gamma(h)$. The boundary condition $W$ is the graph $\Gamma(g)$ of some $g \in U(n)$ and so we require that

$$
g_{0}^{-1} K_{\lambda}(0, y ; W) v=K_{\lambda}(1, y ; W) v
$$

that is,

$$
g_{0}^{-1} \tau_{\lambda}(o) P_{\lambda} \tau_{\lambda}(y)^{-1} v=\tau_{\lambda}(1) Q_{i} \tau_{\lambda}(y)^{-1} v
$$

which implies that

$$
g_{0}^{-1} P_{\lambda}=h_{\lambda}\left(1+P_{\lambda}\right)
$$

Hence

$$
\begin{aligned}
& P_{\lambda}=\left(g_{0}^{-1} h_{\lambda}^{-1}-I\right)^{-1}=\left(e^{\imath \lambda} h^{-1} g_{0}^{-1}-I\right)^{-1} \text { and } \\
& Q_{\lambda}=\left(I-g_{0} h_{\lambda}\right)^{-1}=\left(I-e^{-l \lambda} g_{0} h\right)^{-1} .
\end{aligned}
$$

For $\operatorname{Re}(s)>1$ we now have two formulas for $\zeta_{D_{W}}(s)$; one using $P_{\lambda}$ and one using $Q_{\lambda}$. But only the first of these defines an entire function of $s$, because then $\operatorname{Tr}\left(K_{\lambda}(x, x ; W)\right)=\operatorname{Tr}\left(P_{\lambda}\right) \longrightarrow 0$ exponentially as $\lambda \rightarrow-i \infty$, whereas $\operatorname{Tr}\left(Q_{\lambda}\right) \rightarrow 1$. Thus with $K_{\lambda}(x, y ; W)=\tau_{\lambda}(x) P_{\lambda} \tau_{\lambda}(y)^{-1}$ we have the desired analytic continuation to $C$. Note that inspection of where Seeley [22] says $k_{s}(x, x ; W)$ has poles shows there are no poles for a first order operator.

This means that for $W \in G r_{\text {sso }}$ the derivative of $\zeta_{D_{W}}(s)$ at 0 exists, and so the

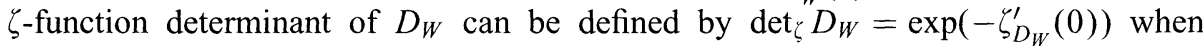
$\operatorname{Ker} D_{W}=0$ and 0 otherwise.

We identify $\operatorname{det}_{\zeta} D_{W}$ precisely in the following way. We have

$$
\begin{aligned}
\zeta_{D_{W}}(s) & =\operatorname{Tr}\left(D_{W}^{-s}\right) \\
& =\frac{1}{2 \pi i} \int_{X} \int_{\gamma} \lambda^{-s} \operatorname{Tr}\left(i K_{\lambda}(x, x ; W)\right) d \lambda d x \\
& =\frac{1}{2 \pi} \int_{\gamma} \lambda^{-s} \operatorname{Tr}\left(\left(e^{i \lambda} h^{-1} g_{0}^{-1}-I\right)^{-1}\right) d \lambda .
\end{aligned}
$$

As a consequence of Cauchy's Theorem the path $\gamma$ of integration may be deformed without affecting the value of the integral so that the rays proceed along the negative axis between $-i \infty$ and $-i \delta$. So with $\lambda=-i \alpha$ and $\alpha \in[0, \infty)$,

$$
\begin{aligned}
\operatorname{Tr}\left(D_{W}^{-s}\right)= & -\frac{1}{2 \pi} \int_{\infty}^{\delta}\left(e^{-\frac{i \pi}{2}} \alpha\right)^{-s} \operatorname{Tr}\left(\left(e^{\alpha} h^{-1} g_{0}^{-1}-I\right)^{-1}\right) d \alpha \\
& -\frac{1}{2 \pi} \delta^{-s+1} \int_{-\frac{\pi}{2}}^{\frac{3 \pi}{2}}\left(e^{l \theta}\right)^{-s+1} \operatorname{Tr}\left(\left(e^{\delta(i \cos \theta-\sin \theta)} h^{-1} g_{0}^{-1}-I\right)^{-1}\right) d \theta \\
& -\frac{1}{2 \pi} \int_{\delta}^{\infty}\left(e^{-\frac{13 \pi}{2}} \alpha\right)^{-s} \operatorname{Tr}\left(\left(e^{\alpha} h^{-1} g_{0}^{-1}-I\right)^{-1}\right) d \alpha
\end{aligned}
$$


Provided $\operatorname{Re}(s) \leqq 1$, as $\delta \rightarrow 0$ then $\left(e^{i \theta}\right)^{-s+1}\left(e^{\delta(l \cos \theta-\sin \theta)} h^{-1} g_{0}^{-1}-I\right)^{-1}$ tends uniformly to its limit, and so we have

$$
\operatorname{Tr}\left(D_{W}^{-s}\right)=-\frac{1}{2 \pi} \int_{0}^{\infty}\left[\left(e^{-\frac{i \pi}{2}} \alpha\right)^{-s}-\left(e^{\frac{13 \pi}{2}} \alpha\right)^{-s}\right] \operatorname{Tr}\left(\left(e^{\alpha} h^{-1} g_{0}^{-1}-I\right)^{-1}\right) d \alpha,
$$

and hence

$$
\begin{aligned}
\zeta_{D_{W}}^{\prime}(0) & =\frac{i}{2 \pi} \int_{0}^{\infty}\left[\log \left(e^{-\frac{i \pi}{2}} \alpha\right)-\log \left(e^{\frac{13 \pi}{2}} \alpha\right)\right] \operatorname{Tr}\left(\left(e^{\alpha} h^{-1} g_{0}^{-1}-I\right)^{-1}\right) d \alpha \\
& =\int_{0}^{\infty} \operatorname{Tr}\left(e^{\alpha} h^{-1} g_{0}^{-1}-I\right)^{-1} d \alpha .
\end{aligned}
$$

From the identity $\delta \log \operatorname{det}(P)=\operatorname{Tr}\left(P^{-1} \delta P\right)$, we obtain

$$
\frac{d}{d \alpha} \log \operatorname{det}\left(I-e^{-\alpha} h g_{0}\right)=\operatorname{Tr}\left(e^{\alpha} h^{-1} g_{0}^{-1}-I\right)^{-1}
$$

and so

$$
\zeta_{D_{W}}^{\prime}(0)=-\log \operatorname{det}\left(I-h g_{0}\right)=-\log \operatorname{det}\left(I-g_{0} h\right) .
$$

And that completes the proof of Theorem (1.3).

The $\eta$-function of $D_{W}$ is defined formally by $\eta_{D_{W}}(s)=\sum_{\lambda} \operatorname{sgn}(\lambda) \lambda^{-s}$, where $\lambda$ runs through the eigenvalues of $D_{W}$. Then for $W \in G r_{\text {iso, } 2 n}$ and $q=\zeta_{\Delta_{W}}(0)$ the relation of the $\zeta$-determinant to the $\zeta$-function norm on the determinant line is given by

\section{Corollary 5.1.}

$$
\operatorname{det}_{\zeta}\left(D_{W}\right)=\exp \left(i \frac{\pi}{2}\left(\eta_{D_{W}}(0)-q\right)\right)\left\|\operatorname{det} D_{W}\right\|_{\zeta}
$$

Proof. Because $\zeta_{D_{W}}(s)$ is entire so is $\zeta_{\Delta_{W}}(s)$. From the identity

$$
\zeta_{D_{W}}(s)=\frac{1}{2}\left(1+e^{-i \pi s}\right) \zeta_{\Delta_{W}}\left(\frac{s}{2}\right)+\frac{1}{2}\left(1-e^{-i \pi s}\right) \eta_{D_{W}}(s),
$$

one has that $\eta_{D_{W}}(s)$ is entire for $\operatorname{Re} s>1$, and elsewhere has at most simple poles. In this 1-dimensional case one has by a similar type of analysis as for the $\zeta$-function, that the residue at 0 vanishes and hence that $\eta_{D_{W}}(0)$ is finite. Differentiating the identity with respect to $s$ and evaluating at zero proves the asserted relation.

Example. Consider $D=i \frac{d}{d x}$ acting on $C^{\infty}\left([0, \pi] ; C^{n}\right)$ and with $W=\Gamma(g)$ for some $g \in U(n)$. Since the eigenvalues of $D_{\Gamma(g)}$ and $D_{\Gamma\left(k^{-1} g k\right)}$ for $k \in U(n)$ coincide we may take $g$ to be diagonal with diagonal entries $u_{j}=e^{2 \pi \imath x}$ for $j=1, \ldots, n$. Then we have that $\operatorname{Spec}\left(D_{W}\right)=\left\{m-\alpha_{j}: m \in Z\right\}$, and so $\zeta_{D_{W}}(s)=\sum_{j=1}^{n} \sum_{m \in Z}\left(m-\alpha_{j}\right)^{-s}$. Thus one way to calculate $\operatorname{det}_{\zeta}\left(D_{\Gamma(g)}\right)$ is by using standard formulas for the Hurwitz $\zeta$-function. The approach we have taken is to rather observe that the integral expression $f(s)=\sum_{j=1}^{n} u_{j} \int_{\gamma} t^{-s}\left(e^{2 \pi t t}-u_{j}\right)^{-1} d t$ defines an analytic continuation of $\zeta_{D_{W}}(s)$ to all of $C$, and so we can evaluate

$$
\zeta_{D_{W}}^{\prime}(0)=\sum_{j=1}^{n}-u_{\jmath} \int_{\gamma} \log t\left(e^{2 \pi \imath t}-u_{j}\right)^{-1} d t=-\log \prod_{j=1}^{n}\left(1+e^{-2 \pi \imath x_{\jmath}}\right),
$$


and hence

$$
\operatorname{det}_{\zeta}\left(D_{\Gamma(g)}\right)=\operatorname{det}\left(1-g_{0}\right) .
$$

5c. The gauge determinant (dimension 1). The 1-dimensional determinant can be calculated more simply, as follows, using intrinsic properties of the determinant line and the fact that $U(n)$ connections on the bundle $\mathscr{E}$ over $X=[0,1]$ are all gauge equivalent to the trivial connection.

More precisely, let $\tau_{0}(x) \in$ End $\left(\mathscr{E}_{1}, \mathscr{E}_{x}\right)$ be the parallel transport matrix at $x \in$ $X$ of the connection $\nabla$ along $X=[0,1]$ from $x=1$. Thus $\nabla_{d / d x} \tau_{0}(x)=0$ with $\tau_{0}(1)=I$ and $\tau_{0}(0)^{-1}=h$, where $K=\Gamma(h)$. By picking a frame for the fibre $\mathscr{E}_{1}$ over $x=1$ we obtain a global trivialisation for $\mathscr{E}$. Hence we may identify $C^{\infty}(X ; \mathscr{E})$ with $C^{\infty}\left(X ; C^{n}\right)$ (the calculation of the determinant is independent of the choice of trivialization of $\mathscr{E}$ ). Moreover, $\nabla$ is gauge equivalent to the trivial connection by the gauge transformation $\tau_{0}(x)$. That is,

$$
d=\tau_{0}^{-1} \nabla \tau_{0} .
$$

Now let $C_{0}^{\infty}\left(X ; C^{n}\right)=\left\{\phi \in C^{\infty}\left(X ; C^{n}\right): \phi(0)=\phi(1)=0\right\}$ and let $D_{0}$ denote the restriction of $D$ to $C_{0}^{\infty}\left(X ; C^{n}\right)$. Then we have the following commutative diagram with exact rows and Fredholm columns:

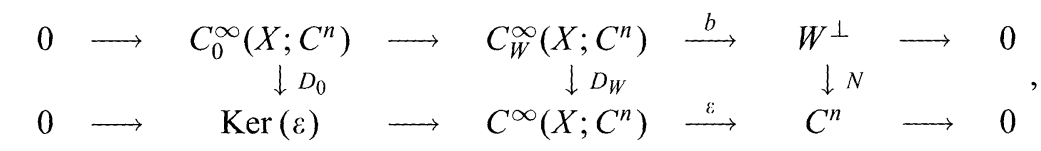

where

$$
\varepsilon(\phi)=\int_{0}^{1} \tau_{0}^{-1}(x) \phi(x) d x, \quad \text { and } \quad N(\alpha, \beta)=i(\beta-h \alpha) .
$$

The commutativity of right-hand square is immediate from (31).

Proposition (4.1) now identifies a canonical isomorphism of determinant lines

$$
\mathscr{L}_{W} \cong \mathscr{L}_{0} \otimes \operatorname{Det}\left(W^{\perp}\right)^{*} \otimes \operatorname{Det} C^{n},
$$

which for invertible $D_{W}$ sends the determinant element $\operatorname{det} D_{W} \in \mathscr{L}_{W}$ to

$$
\operatorname{det} D_{0} \otimes \operatorname{det} N \in \mathscr{L}_{0} \otimes \operatorname{Det}\left(W^{\perp}\right)^{*} \otimes \operatorname{Det} C^{n} .
$$

Moreover, det $D_{0}$ is non-zero and independent of $W$ and so one has

$$
\mathscr{L}_{W} \cong \operatorname{Det}\left(W^{\perp}\right)^{*} \otimes \operatorname{Det} C^{n}=\operatorname{Det} N \text {, }
$$

canonically. But since $W^{\perp}=\left\{\left(g_{0} x, x\right): x \in C^{n}\right\}$, where $g_{0}=-g^{-1} \in U(n)$ and $W=\Gamma(g)$, the right-hand determinant line is canonically trivialized by the projection isomorphism $p r: W^{\perp} \rightarrow C^{n}$ onto the second factor. Thus we have canonical isomorphisms

$$
\mathscr{L}_{W} \cong \operatorname{Det}\left(N \circ p r^{-1}\right) \cong C
$$

which take $\operatorname{det} D_{W}$ to $\operatorname{det} N \circ \mathrm{pr}^{-1}$. Hence we define the gauge determinant $\operatorname{det}_{\mathscr{G}} D_{W}$ of $D_{W}$ by

$$
\operatorname{det}_{\mathscr{G}} D_{W}=\operatorname{det}\left(N \circ p r^{-1}\right) \text {. }
$$

For $v \in C^{n}$ we have by construction that

$$
N \circ p r^{-1}(v)=N\left(\left(g_{0} v, v\right)\right)=i\left(1-h g_{0}\right)(v) \text {, }
$$

and hence the following identification. 
Theorem 5.1. The gauge determinant coincides with the $\zeta$-function determinant and the canonical determinant (without the factor 1/2) up to a factor $i^{n}$. That is,

$$
\operatorname{det}_{\mathscr{G}} D_{W}=i^{n} \operatorname{det}\left(1-g_{0} h\right) .
$$

\section{Proof of Theorem (1.4)}

Let $D_{M}: C^{\infty}\left(M ; S_{M}\right) \rightarrow C^{\infty}\left(M ; S_{M}\right)$ be the first-order elliptic operator over $M=$ $X^{0} \cup_{Y} X^{1}$ constructed from the Dirac operators $D^{0}, D^{1}$ over $X^{0}$ and $X^{1}$ acting on sections of the respective (compatible) spinor bundles $S^{0}, S^{1}$. The canonical determinant $\operatorname{det}_{\mathscr{C}} D_{M}$ of $D_{M}$ is defined by $\operatorname{det}_{\mathscr{C}} D_{M}=\operatorname{det} \mathscr{C}_{M}$, where $\mathscr{C}_{M}$ is the Fredholm operator

$$
\mathscr{C}_{M}: K^{0} \oplus K^{1} \rightarrow F, \quad \mathscr{C}_{M}\left(\phi^{0}, \phi^{1}\right)=\frac{1}{\sqrt{2}}\left(i_{K^{0}}\left(\phi^{0}\right)+i_{K^{1}}\left(\phi^{1}\right)\right)
$$

Here $i_{K^{0}}, i_{K^{1}}$ are the inclusion maps, $K^{0}$ is the graph of $h_{0}: F^{+} \rightarrow F^{-}$and $K^{1}$ is the graph of $h_{1}: F^{-} \rightarrow F^{+}$defined by Theorem (1.1).

Proposition 6.1. $\mathscr{C}_{M}$ is Fredholm and there is a canonical isomorphism of (Quillen) determinant lines

$$
L\left(D_{M}\right) \cong L\left(\mathscr{C}_{M}\right)
$$

which takes $\operatorname{det} D_{M}$ to $\operatorname{det} \mathscr{C}_{M}$ when $D_{M}$ is invertible.

Proof. Let $J\left(Y ; S_{Y}\right)$ denote the space of infinite jets of sections of the boundary spinor bundle $S_{Y}$. An element $\zeta$ of $J\left(Y ; S_{Y}\right)$ has a formal asymptotic expansion $\zeta=\sum_{k} \zeta_{k}(y) \frac{u^{k}}{k !}$, where $\left\{\zeta_{k}\right\}$ is a sequence of smooth sections in $C^{\infty}\left(Y ; S_{Y}\right)$, and $u$ is a coordinate transverse to $Y$ in a tubular neighbourhood of $Y$ in $M$. The proof consists in showing that the following commutative diagram is commutative with exact rows and columns:

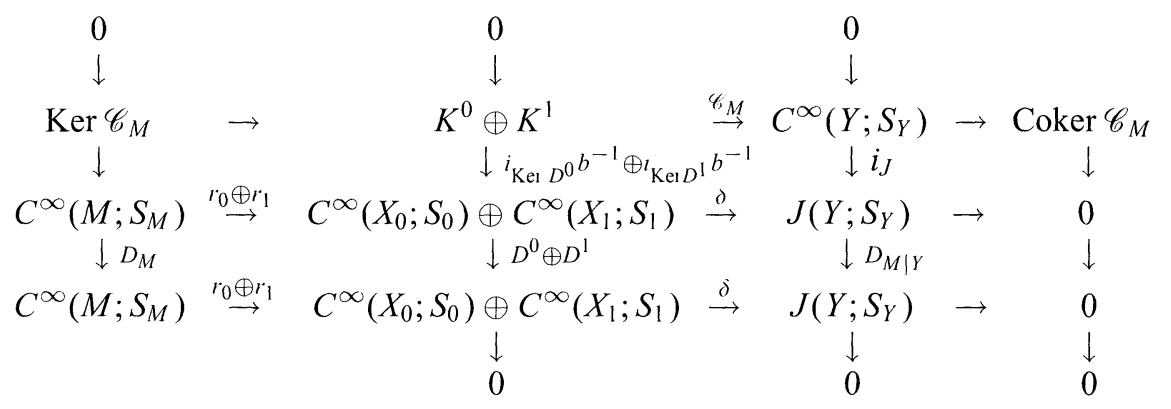

Here $r_{0}, r_{1}$ are the restriction maps, $i_{J}$ is an inclusion map we shall identify below, and $\delta\left(\psi_{0}, \psi_{1}\right)=\sigma J \psi_{0}+J \psi_{1}$, where $J \psi$ denotes the asymptotic expansion near $Y$ of $\psi$ in $u$.

To see that $\operatorname{Ker} \delta \cong C^{\infty}\left(M ; S_{M}\right)$, it is enough to define an injective section of $r_{0} \oplus r_{1}$ over $\operatorname{Ker} \delta$. Recall that two elements $\psi_{k} \in C^{\infty}\left(X_{k} ; S_{k}\right)(k=0,1)$ fit together to give an element of $C^{\infty}\left(M ; S_{M}\right)$ precisely when $\sigma b \psi_{0}$ and $b \psi_{1}$ have the same values and normal derivatives of all orders of $Y$, and that $\sigma: F \rightarrow F$ is an isometry. Hence such a section is defined by $s\left(\psi_{0}, \psi_{1}\right)=\left(\rho\left(\sigma b \psi_{0}\right), \psi_{1}\right)$, where $\rho: F \rightarrow C^{\infty}\left(X_{0} ; S_{0}\right)$ is the map defined in Lemma A.5 (Appendix A). 
For the surjectivity of $\delta$ it is enough to show that the map $C^{\infty}\left(X_{0} ; S_{0}\right) \rightarrow$ $J\left(Y ; S_{Y}\right)$ is surjective and to restrict attention to sections with support contained in a collar neighbourhood $U_{0}=[0,1] \times Y$. But a section $\psi$ on $U_{0}$ with prescribed Taylor series $\sum \psi_{k}(y) \frac{u^{k}}{k !}$ at $u=0$ is given by $\psi(u, y)=\sum \psi_{k}(y) \chi\left(\lambda_{k} u\right) \frac{u^{k}}{k !}$, where $\chi$ is a bump function with $\operatorname{supp}(\chi) \subset U_{0}$ and $\chi=1$ near $Y$, and $\lambda_{k}$ depends on $\psi_{k}$ and tends rapidly to $\infty$.

To see that $\operatorname{Ker} \mathscr{C}_{M} \cong \operatorname{Ker} D_{M}$ we identify $K^{0}$ with $\operatorname{Ker} D^{0}$ and then expand $\psi_{0} \in \operatorname{Ker} D^{0}$ with support contained in $U_{0}$ as $\psi_{0}(u, y)=\sum_{\lambda} e^{-\lambda u} \psi_{0}(0) \phi_{\lambda}(y)$, where $\left\{\phi_{i}\right\}$ are a basis of $F$ of eigenvectors of $A$ with eigenvalues $\lambda$. This follows from (6), and, from the local form $D^{1}=\left(\frac{\partial}{\partial u}+A\right) \sigma$ of $D^{1}$ in the collar of $X^{1}$, we obtain a similar formula for an element of $\operatorname{Ker} D^{1}$. Hence it is enough to require that the zero th order normal derivatives match up to get an element of $\operatorname{Ker} D_{M}$.

To show that the third column is exact we use the fact that in a tubular neighbourhood $V=[-1,1] \times Y$ of $Y$ in $M$, where $Y$ corresponds to $\{0\} \times Y$, the elliptic operator $D_{M}$ splits into a normal and $Y$ component and hence acts on $\zeta \in J\left(Y ; E_{Y}\right)$ as

$$
D_{M \mid Y}(\zeta)=\sum_{k} \sigma\left(\frac{\partial}{\partial u}+A\right) \zeta_{k} \frac{u^{k}}{k !}=\sum_{k} \sigma\left(\zeta_{k+1}+A \zeta_{k}\right) \frac{u^{k}}{k !} .
$$

So $\operatorname{Ker} D_{M \mid Y}$ consists of sequences $\left\{\zeta_{k}\right\}$, defining elements of $J\left(Y ; S_{Y}\right)$, such that $\zeta_{k}=(-1)^{k} A^{k} \zeta_{0}$. That is,

$$
\operatorname{Ker}\left(D_{M \mid Y}\right) \cong C^{\infty}\left(Y ; S_{Y}\right), \quad(-1)^{k} A^{k} \zeta_{0} \leftrightarrow \zeta_{0},
$$

and this defines the inclusion $i_{J}$. Now suppose that $\tau=\sum \tau_{k} \frac{u^{k}}{k !} \in J\left(Y ; S_{Y}\right)$. Then solving iteratively the difference equation $\zeta_{k+1}+A \zeta_{k}=\tau_{k}$, so $\zeta_{0}=0, \zeta_{1}=\tau_{0}, \zeta_{2}=$ $\tau_{1}-A \tau_{0}$, etc., defines an element of $J\left(Y ; S_{Y}\right)$ which $D_{M \mid Y}$ maps to $\tau$. Thus $D_{M \mid Y}$ is surjective and hence the third column is exact.

The commutativity of the diagram is clear except, perhaps, for the top-middle square. To see that it does commute it is enough to consider $\left(\psi_{0}, \psi_{1}\right) \in \operatorname{Ker} D^{0} \oplus$ $\operatorname{Ker} D^{1}$ with support in the tubular neighbourhood $V$ of $Y$, where $\psi_{k}=b^{-1} \phi_{k}$ and $\phi_{k} \in K^{k}$. Here the sections take the form $\psi_{k}(u, y)=\sum_{\lambda} e^{-i u} \psi_{\lambda}^{k}(0) \phi_{\lambda}(y)$. Expanding the exponential term we have

$$
\delta\left(\psi_{0}, \psi_{1}\right)(u, y)=\sum_{\lambda} \sum_{k=0}^{\infty}(-1)^{k} \frac{\lambda^{k}}{\sqrt{2} k !} u^{k}\left(\psi_{i}^{0}(0)+\psi_{\lambda}^{1}(0)\right) \phi_{\lambda}(y) .
$$

But

$$
\begin{aligned}
i_{J} \mathscr{C}_{M}\left(\psi_{0}, \psi_{1}\right)(u, y) & =i_{J}\left(\sum_{\lambda} \frac{1}{\sqrt{2}}\left(\psi_{\lambda}^{0}(0)+\psi_{\lambda}^{1}(0)\right) \phi_{\lambda}(y)\right) \\
& =\sum_{k=0}^{\infty} \sum_{\lambda} \frac{1}{\sqrt{2}}\left(\psi_{\lambda}^{0}(0)+\psi_{\lambda}^{1}(0)\right)(-1)^{k} A^{k} \phi_{\lambda}(y) \frac{u^{k}}{k !},
\end{aligned}
$$

where the final equality is defined by the correspondence (33). Because the $\phi_{\lambda}$ are eigenvectors of $A$, that proves the commutativity.

The Snake Lemma now identifies from the outside columns of the diagram an exact sequence

$$
0 \rightarrow \operatorname{Ker} \mathscr{C}_{M} \rightarrow C^{\infty}\left(M ; S_{M}\right) \stackrel{D_{M}}{\rightarrow} C^{\infty}\left(M ; S_{M}\right) \rightarrow \operatorname{Coker} \mathscr{C}_{M} \rightarrow 0
$$


and therefore canonically identifies $\operatorname{det} D_{M}$ as an element $\operatorname{det}_{\%}, D_{M} \in \wedge\left(\operatorname{Ker} \mathscr{C}_{M}\right)^{*} \otimes$ Coker $\mathscr{C}_{M}=L\left(\mathscr{C}_{M}\right)$. The proof now proceeds in precisely the same way as Proposition (5.1).

To complete the proof of Theorem (1.4) notice that we may now rewrite the top exact sequence of the diagram as

$$
0 \rightarrow \operatorname{Ker} D_{M} \rightarrow K^{0} \oplus K^{1} \stackrel{4, M}{\rightarrow} F \rightarrow \text { Coker } D_{M} \rightarrow 0
$$

and so if $D_{M}$ is invertible then $\mathscr{C}_{M}$ is an isomorphism. In precisely the same way as in Sect. 5, we obtain

$$
\operatorname{det}_{,}, D_{M}=\operatorname{det} \frac{1}{\sqrt{2}}\left(\begin{array}{cc}
1 & h_{1} \\
h_{0} & 1
\end{array}\right)
$$

and hence, as in Sect. 5 , there is the canonical identification $\operatorname{det}_{\%}, D_{M}=\operatorname{det} \frac{1}{2}(1-$ $\left.h_{1} h_{0}\right)$, which is a well-defined complex number because $(1 / 2)\left(1-h_{0} h_{1}\right)$ is of the form $1+$ smoothing.

\section{Proof of Theorem (1.5)}

We assume that the Hermitian bundle $\mathscr{E}_{S}$ has been trivialized so that the isotropic Grassmannians of self-adjoint boundary conditions for $D^{0}$ and $D^{1}$ may be identified as $G r_{150.2 n} \cong U(n)$. Then from Theorems (1.2) and (1.4) the identity we are to prove may be rewritten as

$$
\operatorname{det}\left(1-h_{1} h_{0}\right)=\int_{U(n)} \operatorname{det}\left(1-g_{0} h_{0}\right) \operatorname{det}\left(1-g h_{1}\right) d g
$$

(When $n=1$, this is just the identity $1-e^{l(y+\beta)}=\int_{0}^{2 \pi}\left(1+e^{l(y-l)}\right)\left(1-e^{l(\beta+0)}\right)$ $d 0 / 2 \pi$.)

We do that as follows. Let $\operatorname{End}\left(\wedge C^{n}\right)$ denote the space of endomorphisms of the exterior algebra $\wedge C^{n}=\bigoplus_{k=0}^{n} \wedge^{k} C^{n}$, with the standard Hermitian inner-product

$$
(,): \text { End }\left(\wedge C^{n}\right) \otimes \operatorname{End}\left(\wedge C^{n}\right) \rightarrow C, \quad\left(T_{0}, T_{1}\right)=\operatorname{Tr}\left(T_{0}, T_{1}^{*}\right) .
$$

Let $\varepsilon \in \operatorname{End}_{0}\left(\wedge C^{n}\right)$ be the operator equal to $(-1)^{k}$ on $\wedge^{k} C^{n}$. Then one has the supertrace

$$
\operatorname{Tr}_{s}: \text { End }\left(\wedge C^{n}\right) \rightarrow C, \quad \operatorname{Tr}_{s}(T)=\operatorname{Tr}(\varepsilon T) .
$$

On elements $T$ in the space $\operatorname{End}_{0}\left(\wedge C^{n}\right)$ of degree preserving endomorphisms

We define a map

$$
\operatorname{Tr}_{s}(T)=\sum_{k=1}^{n}(-1)^{h} \operatorname{Tr}\left(T_{\mid \wedge^{h} C^{\prime \prime}}\right) \text {. }
$$

$$
\operatorname{End}_{0}\left(\wedge C^{n}\right) \rightarrow L^{2}(U(n)), \quad T \mapsto f_{T},
$$

where $f_{T}(g)=\operatorname{Tr}_{s}\left(T \circ \wedge g_{0}\right)$. The map (38) is an isometry, that is,

$$
\operatorname{Tr}\left(T_{0} T_{1}^{*}\right)=\int_{U(n)} f_{T_{0}}(g) \overline{f_{T_{1}}(g)} d g .
$$

To see that, notice that by the Peter-Weyl theorem $L^{2}(U(n))$ is an irreducible unitary representation of $U(n) \times U(n)$, as is $\operatorname{End}_{0}\left(\wedge C^{n}\right) \cong \bigoplus_{k} \wedge^{k}\left(C^{n}\right)^{*} \otimes \wedge^{k}\left(C^{n}\right)$. 
Because the map is bi-invariant and not zero (evaluation at the identity) then by Schur's lemma it is an isomorphism which preserves the $U(n)$-invariant metric.

Applying (39) to the elements $\wedge h_{0}, \wedge-h_{1}^{-1} \in \operatorname{End}_{0}\left(\wedge C^{n}\right)$ we obtain

$$
\begin{aligned}
\operatorname{Tr}\left(\wedge h_{0} \wedge\left(-h_{1}^{-1}\right)^{*}\right) & =\int_{U(n)} \operatorname{Tr}_{s}\left(\wedge h_{0} \circ \wedge g_{0}\right) \overline{\operatorname{Tr}_{s}\left(\wedge-h_{1}^{-1} \circ \wedge g_{0}\right)} d g \\
& =\int_{U(n)} \operatorname{Tr}_{s}\left(\wedge h_{0} \circ \wedge g_{0}\right) \operatorname{Tr}_{s}\left(\left(\wedge-h_{1}^{-1} \circ \wedge g_{0}\right)^{*}\right) d g \\
& =\int_{U(n)} \operatorname{Tr}_{s}\left(\wedge\left(h_{0} g_{0}\right)\right) \operatorname{Tr}_{s}\left(\wedge\left(h_{1} g\right)\right) d g \\
& =\int_{U(n)} \operatorname{det}\left(1-g_{0} h_{0}\right) \operatorname{det}\left(1-g h_{1}\right) d g
\end{aligned}
$$

The final equality follows since

$$
\operatorname{Tr}_{s}\left(\wedge\left(h_{0} g_{0}\right)\right)=\operatorname{Tr}_{s}\left(\bigoplus_{k=0}^{n} \wedge^{k}\left(h_{0} g_{0}\right)\right)=\sum_{k=0}^{n}(-1)^{k} \operatorname{Tr}\left(\wedge^{k}\left(h_{0} g_{0}\right)\right)=\operatorname{det}\left(1-g_{0} h_{0}\right)
$$

and similarly for the second factor in the integrand. Because

$$
\operatorname{Tr}\left(\wedge h_{0} \wedge\left(-h_{1}^{-1}\right)^{*}\right)=\sum_{k=0}^{n}(-1)^{k} \operatorname{Tr}\left(\wedge^{k}\left(h_{0} h_{1}\right)\right)=\operatorname{det}\left(1-h_{1} h_{0}\right)
$$

that completes the proof.

\subsection{Concluding remarks on the relation with $0+1$-dimensional TQFT}

The choice of the elements $\wedge h_{0}, \wedge-h_{1}^{-1} \in \operatorname{End}_{0}\left(\wedge C^{n}\right)$ in the proof of Theorem (1.5) is not arbitrary. This becomes apparent when the theorem is realized as identifying the canonical pairing of a $0+1$-dimensional topological quantum field theory. That is explained in detail in [20], where determinants of boundary value problems arise as terms in the evaluation of the Feynman path integral defining the theory. However, it may be useful to make a few brief remarks here. A $d+1$-dimensional TQFT is characterized in $[2,26]$ as a functor $Z$ which assigns to each $d$-dimensional manifold $Y$ a vector space $Z(Y)$ and to each $d+1$-dimensional manifold $X$ with boundary $Y$ a vector $Z_{X} \in Z(Y)$, and which satisfies certain natural axioms. The most important of these is the "sewing axiom" which requires for a closed $d+1$-dimensional manifold $M=X^{0} \cup_{Y} X^{1}$ that

$$
Z_{M}=\left\langle Z_{X^{0}}, Z_{X^{1}}\right\rangle
$$

where $\langle\rangle:, Z(Y) \otimes Z(\bar{Y}) \rightarrow C$ is the canonical bilinear pairing arising from the "duality axiom" $Z(\bar{Y})=Z(Y)^{*}$ and $\bar{Y}$ denotes $Y$ with the opposite orientation. For $d=0$ and $M=S^{1}$ we naturally obtain such a structure by. identifying $Z_{X^{k}}$ with $\operatorname{det} D^{k}$, for $k=0,1$, where the $D^{k}$ are as in Theorem (1.5). Thus $Z_{X^{0}}, Z_{X^{1}}$ arise as elements of the Fock spaces associated to the holomorphic determinant line bundles $L^{0}, L^{1}$ over the "classical phase space" $G r_{2 n}$. Moreover, the canonical Hermitian connection on $L^{0}$ defined by the $\zeta$-function metric has curvature $i \omega$, where $\omega$ is the Kähler form on $G r_{2 n}$ [20]. Hence the dual line bundle $\left(L^{0}\right)^{*}$ is a quantum line bundle in the sense of Kähler quantization [29], through which 
one obtains $Z(\{0,1\})=\Gamma_{\text {hol }}\left(G r_{2 n} ;\left(L^{0}\right)^{*}\right)^{*}$ as the Hilbert space of the theory. Here the boundary $Y$ of $X^{0}$ is taken to be $\{0,1\}$. However, we know from Proposition (4.4) that $L^{0} \cong \operatorname{Det} \xi \otimes \operatorname{Det}\left(\mathscr{K}^{0}\right)^{*}$, while it is well known [17](p. 22) that there is a canonical isomorphism $\Gamma_{\text {hol }}\left(G r_{2 n}\right.$; Det $\left.\xi^{*}\right) \cong \wedge\left(C^{2 n}\right)^{*}$. Hence there are canonical isomorphisms

$$
\begin{aligned}
Z(\{0,1\}) & \cong \wedge C^{2 n} \otimes\left(\operatorname{Det} K^{0}\right)^{*} \\
& \cong \wedge C^{2 n} \otimes\left(\operatorname{Det} C^{n}\right)^{*},
\end{aligned}
$$

where the latter isomorphism depends on the fact that $K^{0}=\Gamma\left(h_{0}\right)$. Mathematically the $0+1$-dimensional TQFT comes down to the Borel-Weyl theorem for the unitary group, as predicted by Atiyah [2]. More precisely, because $C^{2 n}=C^{n} \oplus C^{n}$ relative to the boundary points 0,1 choosing an element $\lambda$ in the second factor in (40) defines an isomorphism $Z(\{0,1\}) \cong$ End $\left(\wedge C^{n}\right)$. The identification $K^{0}=\Gamma\left(h_{0}\right)$ gives a preferred choice for $\lambda$ and the corresponding isomorphism takes $\operatorname{det} D^{0}$ to $\wedge h_{0}$. The bilinear pairing evaluated on the determinant sections

$$
\langle,\rangle: \text { End }\left(\wedge C^{n}\right) \otimes \operatorname{End}\left(\wedge C^{n}\right) \rightarrow C,
$$

thus coincides with (37), and the pairing of the TQFT is the identity

$$
\operatorname{det} D_{M}=\left\langle\operatorname{det} D^{0}, \operatorname{det} D^{1}\right\rangle,
$$

which from the point of view of the representation theory may be seen as a character formula. Proofs of these facts are given in [20].

\section{Appendix A: Construction of a Parametrix}

In this appendix we construct a parametrix for a Dirac boundary value problem $D_{W}$ and we explain the sense in which $D_{W}$ depends holomorphically on $W$.

A1. The parametrix. We build a parametrix for $D_{W}$ in the following way. Let $X^{-}$ denote the manifold $X$ endowed with the reverse orientation and let $M=X \cup_{Y} X^{-}$ be the closed double manifold with "spinor" bundle $S_{M}$ constructed as in Sect. 1.

The Sobolev spaces $H^{r_{1}}\left(M ; S_{M}\right), H^{r_{2}}\left(Y ; S_{Y}\right)$ for the closed manifolds $M$ and $Y$ are defined as usual for any real numbers $r_{1}, r_{2}$. For $X$ we define the Sobolev space $H^{k}(X ; S)$ for each non-negative integer $k$ as the Hilbert space completion of $C^{\infty}(X ; S)$ in the norm

$$
\|\psi\|_{k}^{2}=\sum_{j=0}^{k} \int_{X}\left|\nabla^{J} \psi(x)\right|^{2} d x .
$$

Lemma A1. Let $k$ be a positive integer.

(i) The restriction map $b: C^{\infty}(X ; S) \rightarrow F$ extends to a continuous linear map $b_{k}: H^{k}(X ; S) \rightarrow H^{k-1 / 2}\left(Y ; S_{Y}\right)$. There is a continuous linear section $\rho: F \rightarrow C^{\infty}$ $(X ; S)$ for $b$ which extends to a continuous section $\rho_{k}: H^{k-1 / 2}\left(Y ; S_{Y}\right) \rightarrow H^{k}(X ; S)$ of $b_{k}$.

(ii) The map $r: C^{\infty}\left(M ; S_{M}\right) \rightarrow C^{\infty}(X ; S)$ restricting smooth sections of $S_{M}$ to the compact submanifold $X$ extends to a continuous linear map $r_{k}: H^{k}\left(M ; S_{M}\right) \rightarrow$ $H^{k}(X ; S)$. There is a continuous linear section $\varepsilon$ of $r$ which extends to a continuous linear section $\varepsilon_{k}: H^{k}(X ; S) \rightarrow H^{k}\left(M ; S_{M}\right)$ of $r_{k}$. 
The existence of the linear section $\varepsilon$ for $r$ is a delicate fact proved by Seeley in [21]. An explicit $\rho$ is constructed in Proposition (A1). For the remaining assertions we refer to [16].

In particular, this implies that for each integer $k>\operatorname{dim} X / 2$ there is a continuous inclusion $H^{k+r}(X ; E) \rightarrow C^{r}(X ; E)$ (Sobolev theorem). For the inclusion factors as

$$
H^{k+r}(X ; E) \stackrel{\varepsilon_{k+r}}{\rightarrow} H^{k+r}\left(M ; E_{M}\right) \rightarrow C^{r}\left(M ; E_{M}\right) \stackrel{r}{\rightarrow} C^{r}(X ; E)
$$

where the central map is given by Sobolev's theorem for a closed manifold, and all the maps involved are continuous. Similarly, one has that for integers $k_{1}<k_{2}$ the inclusion $H^{k_{2}}(X ; E) \rightarrow H^{k_{1}}(X ; E)$ is compact (Rellich lemma). Consequently, $C^{\infty}(X ; S)=H^{\infty}(X ; S)$ and the inverse limit topology on $H^{\infty}(X ; S)$ is the $C^{\infty}$ topology.

By a smoothing operator $C^{\infty}(X ; S) \rightarrow C^{\infty}(X ; S)$ on the manifold $X$ with boundary we mean an operator with continuous extension $H^{k_{1}}(X ; S) \rightarrow H^{k_{2}}(X ; S)$ for all non-negative integers $k_{1}$ and $k_{2}$, and which, by Sobolev's theorem, therefore has image in $C^{\infty}(X ; S)$. Let $O P_{-\infty}(X ; S)$ denote the space of all such operators.

Proposition A1. Let $W$ be in Gr. Then there is a $C^{\infty}$ continuous linear operator

$$
K_{W}: C^{\infty}(X ; S) \rightarrow C_{W}^{\infty}(X ; S)
$$

such that

(i) $K_{W}$ extends to a continuous operator $K_{W}^{l}: H^{l}(X ; S) \rightarrow H^{l+1}(X ; S)$ for each non-negative integer $l$.

(ii) $D_{W} K_{W}-I=R_{1} \in O P_{-\infty}(X ; S)$.

(iii) $K_{W} D_{W}-I=R_{2} \in O P_{-\infty}(X ; S)$.

Proof. First, we recall from elliptic theory on closed manifolds that the doubled operator $D_{M}: C^{\infty}\left(M ; S_{M}\right) \rightarrow C^{\infty}\left(M ; S_{M}\right)$, has a parametrix $\mathscr{K}$ of order -1 , so that $D_{M} \mathscr{K}-I_{M}=Q_{1}$ and $\mathscr{K} D_{M}-I_{M}=Q_{2}$ are smoothing operators on $C^{\infty}\left(M ; S_{M}\right)$, and $\mathscr{K}$ extends to a continuous operator $H^{l}\left(M ; S_{M}\right) \rightarrow H^{l+1}\left(M ; S_{M}\right)$.

An explicit continuous linear section $\rho: F \rightarrow C^{\infty}(X ; S)$ for $b$ is given by $\rho(\phi)(u, y)=\chi(u) e^{-u\left(A^{+} \oplus-A^{-}\right)} \phi(y)$, where the boundary operator is written $A=$ $A^{+} \oplus A^{-}$relative to the energy polarization of $F$, and $\chi$ is a $C^{\infty}$ bump function on $R^{1}$ with $\chi(u)=1$ for $0 \leqq u \leqq \frac{1}{2}$ and $\chi(u)=0$ for $u \geqq 1,(\partial X=Y \times\{0\})$.

. Let $W \in G r$. We define a parametrix $K_{W}$ for $D_{W}$ by

$$
K_{W}=r \mathscr{K} \varepsilon-\rho P_{W} b r \mathscr{K} \varepsilon .
$$

Notice that for $\psi \in C^{\infty}(X ; S)$ one has $P_{W} b K_{W} \psi=0$, so that $K_{W}$ has the correct range. Moreover, by our preliminary remarks the operators defining $K_{W}$ are continuous with a combined order of -1 . That proves (i).

To see (ii) first observe that since $W$ and $H^{+}$are in $G r$ one has that $P_{W}-P^{+}=$ $R_{W}: F \rightarrow F$ is a smoothing operator. For $\phi \in F$ the support of $\rho(\phi)$ lies in the collar neighbourhood $U$, and so for $\psi \in C^{\infty}(X ; S)$,

$$
\begin{aligned}
D_{W} K_{W} \psi & =\operatorname{Dr} \mathscr{K} \varepsilon(\psi)-\sigma\left(\frac{\partial}{\partial u}+A\right) \rho\left(P_{W} \mathscr{E} \psi\right) \quad(\mathscr{E}=b r \mathscr{K} \varepsilon) \\
& =I \psi+r Q_{1} \varepsilon \psi-\sigma\left(\frac{\partial}{\partial u}+A\right) \chi e^{-u\left(A^{+} \oplus-A^{-}\right)}\left(P^{+}+R_{W}\right) \mathscr{E} \psi
\end{aligned}
$$


It remains to explain why the third term is smoothing. Away from the boundary this is immediate because $A^{+} \oplus-A^{-}: F \rightarrow F$ is a positive elliptic operator and hence the heat operator $e^{-u\left(A^{+} \oplus-A^{-}\right)}$is smoothing. Thus for $u>0$ there is a smooth extension of $\mathscr{E} \psi \in H^{k-1 / 2}\left(Y ; S_{Y}\right)$ into the interior of $X$. To see that it is also smooth over the boundary $(u=0)$ we work in the neighbourhood of $Y$ with $u \in[0,1 / 4)$ where $\chi=1$. Because $R_{W}$ is a smoothing operator it is enough to consider just the positive energy part $P^{+} \mathscr{E} \psi$ of $\mathscr{E} \psi$. But $\tau_{u}=e^{-u\left(A^{+} \oplus-A^{-}\right)} P^{+} \xi=e^{-u A^{+}} P^{+} \xi$ and for $0<u<1 / 4$ this is in $\operatorname{Ker} D$. So there is a smooth sequence of sections $\tau_{u} \rightarrow P^{+} \xi$ as $u \rightarrow 0$ with $\left\|D \tau_{u}\right\|_{k}=0$ for $u \in(0,1 / 4)$ and each non-negative integer $k$. By continuity then $\left\|D \tau_{0}\right\|_{k}=0$. That proves (ii). Notice that from this construction it is clear that if $D_{M}$ is invertible the Atiyah-Patodi-Singer problem $D_{H^{+}}$has an exact parametrix $\left(R_{1}=0\right)$ as in [3].

Part (iii) is immediate from the observation that the operator $\left(\varepsilon D-D_{M} \varepsilon\right) r$ : $C^{\infty}\left(M ; S_{M}\right) \rightarrow C^{\infty}\left(M ; S_{M}\right)$ has support contained in the collar neighbourhood of the boundary in $X^{-}$, and that $\mathscr{K}$ preserves supports on $M$ up to a smoothing operator.

Corollary A1. There exists a constant $C$ such that for $\psi \in C_{W}^{\infty}(X ; S)$

$$
\|\psi\|_{k+1} \leqq C\left(\left\|D_{W} \psi\right\|_{k}+\|\psi\|_{k}\right) .
$$

Let $\mathscr{D}_{W}$ denote the $L^{2}$ extension of the operator $D_{W}$. Then the elliptic estimate (43) implies that if $\psi \in \operatorname{dom} \mathscr{D}$ then $\psi \in H^{1}(X ; S)$ and $P_{W} b \psi=0$, where $P_{W}$ and $b$ refer to their Hilbert space extensions.

Conversely, if $\psi \in H_{W}^{1}(X ; S) \stackrel{\text { def }}{=}\left\{\psi \in H^{1}(X ; S): P_{W} b \psi=0\right\}$ then $\psi \in$ dom $\left(\mathscr{D}_{W}^{1}\right)$. For it is sufficient to consider $\psi$ supported on the collar, and show that there $\psi$ is approximated by smooth sections which satisfy the boundary condition. That follows by applying the smoothing operator $e^{-\beta A^{2}}$ in the $Y$ direction, and in the normal direction by extending $\psi$ into a tubular neighbourhood of $Y$ in $M$ by reflection and then smoothing out by convolution. Thus we have

Lemma A2. $\operatorname{dom}\left(\mathscr{D}_{W}\right)=H_{W}^{1}(X ; S)$.

The existence of the parametrix $K_{W}$ means that $D_{W}$ and $D_{W^{*}}$ and their $L^{2}$ extensions are Fredholm operators and that $\operatorname{ker} \mathscr{D}_{W}=\operatorname{ker} D_{W}$ [16]. A particular consequence of the following proposition is that the index of $D_{W}$ can be computed in $C^{\infty}$ or $L^{2}$. The next proposition is well-known $[8,23]$.

Proposition A2. Let $W \in G r$. Then the $L^{2}$ closures of $D_{W}$ and $D_{W^{*}}$ are adjoints of each other.

Proof. From Proposition (A1) we obtain orthogonal decompositions

$$
\operatorname{dom}\left(D_{W}\right)=C_{W}^{\infty}(X ; S)=\operatorname{Ker} D_{W} \oplus N,
$$

and $C^{\infty}(X ; S)=\operatorname{Ker}\left(D_{W^{*}}\right) \oplus \operatorname{Im}\left(D_{W}\right)$, where $N=\left(\operatorname{Ker} D_{W}\right)^{\perp} \cap C_{W}^{\infty}(X ; S)$. The restriction of $D_{W}$ to $N$ is invertible and hence it has a continuous linear inverse $\operatorname{Im}\left(D_{W}\right) \rightarrow N$ which we extend by zero to an operator $T_{W}$ defined on all of $C^{\infty}(X ; S)$. Let $B_{0}$ and $B_{1}$ respectively denote the $L^{2}$ projections onto $\operatorname{Ker} D_{W}$ and $\operatorname{Ker} D_{W^{*}}$. Then, by construction, there are the equalities

$$
D_{W} T_{W}-I=-R_{1}, \quad T_{W} D_{W}-I=-R_{0}, \quad B_{0} T_{W}=0=T_{W} B_{1} .
$$


Repeating this construction with $W^{*}$ yields corresponding equalities for $T_{W *}$, and since $B_{0}$ and $B_{1}$ are smoothing, then $T_{W}$ is a parametrix for $D_{W}$ and $T_{W^{*}}$ is a parametrix for $D_{W^{*}}$. Moreover, the combined equalities imply that $\left\langle\xi, T_{W} \eta\right\rangle_{S}=$ $\left\langle T_{W^{*}} \xi, \eta\right\rangle_{S}$ for $\xi, \eta \in C^{\infty}(X ; S)$. Hence $T_{W}$ and $T_{W^{*}}$ are formal adjoints in $C^{\infty}$. Then by the continuity of the inner-product $\left(\bar{T}_{W}\right)^{*}=\bar{T}_{W^{*}}$.

We have the immediate corollary of Proposition (A2) that if $W$ is a self-adjoint boundary condition then the $L^{2}$ extension $\mathscr{D}_{W}$ is self-adjoint. It is also a straightforward consequence that if $W \in G r$ is a self-adjoint boundary condition then $D_{W}$ is essentially self-adjoint, that is, $D_{W}$ has a unique self-adjoint extension. These facts imply the spectral theorem Proposition (2.1) for elliptic self-adjoint Dirac boundary value problems. The details are now no different to the case for closed manifolds given, for example, in [19]. The parametrix $K_{W} K_{W^{*}}$ for the Laplacian $\Delta_{W}$, where $K_{W}$ and $K_{W^{*}}$ are the parametrices for $D_{W}$ and $D_{W^{*}}$, implies the corresponding spectral theorem for $\Delta_{W}$.

A2. Holomorphic Families. We refer to a family of linear operators $T_{a}: E_{a} \rightarrow$ $F_{a}, a \in \mathscr{A}$ acting between complete Hausdorff locally convex topological vector spaces endowed with continuous norms as a holomorphic family in the sense of [25]. That is, one requires $\mathscr{E}=\cup_{a} E_{a}$ and $\mathscr{F}=\cup_{a} F_{a}$ to be holomorphic vector bundles over $\mathscr{A}$, in the sense of [24], and that there exists a parametrix $S_{a}: F_{a} \rightarrow E_{a}$ such that the family of operators $S_{a} T_{a}-I: E_{a} \rightarrow E_{a}$ and $T_{a} S_{a}-I: F_{a} \rightarrow F_{a}$ are compact operators and continuous with respect to $a$ in the uniform topology. We refer to [25] for further discussion.

Let us, for example, consider the family of boundary value problems

$$
D_{W}: C_{W}^{\infty}(X ; S) \rightarrow C^{\infty}(X ; S), W \in G r .
$$

In this case we take

$$
\mathscr{E}=\bigcup_{W} C_{W}^{\infty}(X ; S) \text { and } \mathscr{F}=G r \times C^{\infty}(X ; S) .
$$

The space $\mathscr{F}$ is trivially a holomorphic vector bundle, while the holomorphic bundle structure on $\mathscr{E}$ is given by the same argument as [24](p. 389). For the parametrix we take $K_{W}: C^{\infty}(X ; S) \rightarrow C_{W}^{\infty}(X ; S)$ as in Proposition A1, and from that proposition we know that $D_{W} K_{W}-I$ and $K_{W} D_{W}-I$ are compact operators. By inspection (from Proposition A1) one sees that the continuity dependence on $W$ of these operators is simply the orthogonal projection operator $P_{W}$ and hence Dirac boundary value problems parameterized by the restricted Grassmannian form a holomorphic family.

\section{Appendix B: Proof of Theorem (4.1)}

Our goal is to prove that there is a canonical isomorphism

$$
\operatorname{Det} D_{M} \cong \mathscr{L}_{W}^{0} \otimes \mathscr{L}_{W \perp}^{1},
$$

which varies smoothly with $W$. Most of the work needed to prove this was done in the proof of Proposition (6.1), we just need the following identifications.

Proposition B1. There is a canonical isomorphism of determinant lines

$$
\operatorname{Det} D_{W \perp}^{1} \cong \operatorname{Det} \rho_{K^{1}}\left(W^{\perp}\right)
$$


depending smoothly on $W$, where $\rho_{K^{1}}\left(W^{\perp}\right)=P_{W^{\perp}} \circ i_{K^{1}}$. If $D_{W \perp}^{1}$ is invertible $\operatorname{det} D_{W \perp}^{1}$ maps to $\operatorname{det} \rho_{K^{1}}\left(W^{\perp}\right)$.

This is just Proposition (4.3) restated for the opposite Grassmannian and so we omit the proof.

Let $\mathscr{C}_{M}: K^{0} \oplus K^{1} \rightarrow F$ be defined by $\mathscr{C}_{M}\left(\phi_{0}, \phi_{1}\right)=i_{K^{0}}\left(\phi_{0}\right)+i_{K^{1}}\left(\phi_{1}\right)$, as in Sect. 6.

Proposition B2. For each $W$ in $G r$ there is a canonical isomorphism of determinant lines

$$
\operatorname{Det} \mathscr{C}_{M} \cong \mathscr{L}_{W}^{0} \otimes \mathscr{L}_{W \perp}^{1}
$$

varying smoothly with $W$. If $W=K^{0}$ the isomorphism preserves the determinant elements.

Proof. First we modify $\mathscr{C}_{M}$ to the map

$$
\mathscr{C}_{w}: K^{0} \oplus K^{1} \rightarrow F, \quad \pi\left(\phi_{0}, \phi_{1}\right)=\rho_{K^{0}}(W)\left(\phi_{0}\right)+i_{K^{1}}\left(\phi_{1}\right) .
$$

There is the following commutative diagram with exact rows:

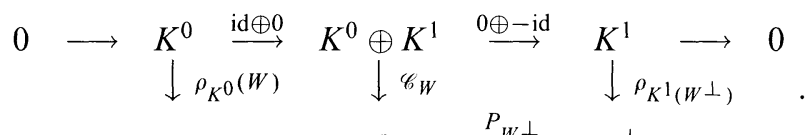

$$
\begin{aligned}
& 0 \longrightarrow W \longrightarrow F \quad \stackrel{P_{W \perp}}{\longrightarrow} W^{\perp} \longrightarrow 0
\end{aligned}
$$

Since $W, K^{0} \in G r$ and $W^{\perp}, K^{1} \in G r^{-}$, all the columns are Fredholm, and so there is a canonical isomorphism depending smoothly on $K^{0}, K^{1}$ and $W$,

$$
\operatorname{Det} \mathscr{C}_{W} \cong \operatorname{Det} \rho_{K^{0}}(W) \otimes \operatorname{Det} \rho_{K^{1}}\left(W^{\perp}\right),
$$

and from Propositions (4.3) and (B1)

$$
\cong \mathscr{L}_{W}^{0} \otimes \mathscr{L}_{W \perp}^{1} \text {. }
$$

If the Dirac operators $D_{W}^{0}, D_{W \perp}^{1}, D_{M}$ are invertible the determinant elements $\operatorname{det} \mathscr{C}_{W}$ and $\operatorname{det} \rho_{K^{0}}\left(W^{\perp}\right) \otimes \operatorname{det} \rho_{K^{1}}\left(W^{\perp}\right)$ correspond under the isomorphism.

One has

$$
\mathscr{C}_{M}-\mathscr{C}_{W}=\left(P_{W \perp} \circ i_{K^{0}}\right) \oplus 0,
$$

and since $W$ and $K^{0}$ are in $G r$ then $P_{W \perp} \circ i_{K^{0}}: K^{0} \rightarrow W^{\perp}$ is smoothing, and hence

$$
\operatorname{Det} \mathscr{C}_{M}=\operatorname{Det} \mathscr{C}_{W} \text {. }
$$

Because $\mathscr{C}_{M}=\mathscr{C}_{W}$ when $W=K^{0}$, we see that the determinant elements then map to each other.

We know from Proposition (6.1) that there is a canonical isomorphism preserving determinant elements

$$
\text { Det } D_{M} \cong L\left(\mathscr{C}_{M}\right) \text {, }
$$

and so that completes the proof.

Acknowledgements. I would in particular like to thank Graeme Segal who suggested many of the ideas presented in this paper. I have also benefited greatly from conversations with S.K. Donaldson, N.F. Johnson, D.G. Quillen and I thank them. I would also like to thank the referee for a helpful critique of this paper. 


\section{References}

1. Atiyah, M.F.: Circular symmetry and stationary phase approximation. Colloquium in honour of Laurent Schwartz, Asterique No. 131, Vol. 1, 43-59 (1989)

2. Atiyah, M.F.: Topological quantum field theories. Publ. Math. I.H.E.S. Paris 68, 175-186 (1989)

3. Atiyah, M.F., Patodi, V.K., Singer, I.M.: Spectral asymmetry and Riemannian geometry: I. Math. Proc. Camb. Phil. Soc. 77, 43-69 (1975)

4. Bismut, J.M., Cheeger, J.: Remarks on the index theorem for families of Dirac operators on a manifold with boundary. Differential Geometry. A Symposium in Honour of Manfred de Carmo. New York: Longman Scientific and Technical, (1991)

5. Bismut, J.M., Freed, D.S.: The analysis of elliptic families: (I) Metrics and connections on determinant bundles, (II) Dirac operators, eta invariants, and the holonomy theorem. Commun. Math. Phys. 106, 159-76 (1986); 107, 103-63 (1986)

6. Booss, B., Bleecker, D.D.: Topology and Analysis. Berlin, Heidelberg, New York: Springer, 1985

7. Booss-Bavnbek, B., Wojciechowski, K.P.: Pseudodifferential projections and the topology of certain spaces of elliptic boundary value problems. Commun. Math. Phys. 121, 1-9 (1989)

8. Booss-Bavnbek, B., Wojciechowski, K.P.: Elliptic boundary value problems for Dirac operators. Basel-Boston: Birkhauser, 1993

9. Burghelea, D., Friedlander, L., Kappeler, T.: On the determinant of elliptic differential and finite difference operators in vector bundles over $S^{1}$. Commun. Math. Phys. 138, 1-18 (1991)

10. Douglas, R.G., Wojciechowski, K.P.: Adiabatic limits of the $\eta$-invariants. The odd-dimensional Atiyah-Patodi-Singer problem. Commun. Math. Phys. 142, 139-68 (1989)

11. Forman, R.: Functional determinants and geometry. Inv. Math. 88, 447-493 (1987)

12. Gilkey, P.B.: Invariance Theory, The Heat Equation, And The Atiyah-Singer Index Theorem. Berkeley, Ca.: Publish or Perish 1984

13. Grubb, G., Seeley, R.T.: Preprint (1993)

14. Lesch, M., Wojciechowski, K.P.: On the $\eta$-invariant of generalized Atiyah-Patodi-Singer problems. Preprint (1993)

15. Maclane, S.: Categories for the working mathematician. Berlin, Heidelberg, New York: Springer, GTM 5, 1971

16. Palais, R.S.: Seminar on the Atiyah-Singer Index Theorem. Princeton, NJ: Princeton Univ. Press, 1965

17. Pressley, A.N., Segal G.B.: Loop Groups, Oxford: Oxford Univ. Press, 1988

18. Quillen, D.G.: Determinants of Cauchy-Riemann operators over a Riemann surface. Funk. Anal. i ego Prilozhenya 19, 37-41 (1985)

19. Roe, J.: Elliptic Operators, Topology and Asymptotic Methods. Essex, England: Longman Scientific and Technical, 1988

20. Scott, S.G.: Determinants, Grassmanians and Functorial QFT. Preprint (1995)

21. Seeley, R.T.: Extension of $C^{\infty}$ functions in a half space. Proc. Amer. Math. Soc. 15, 625-626 (1964)

22. Seeley, R.T.: Complex powers of elliptic operators. Proc. Symp. on Singular Integrals, AMS 10, 288-307 (1976)

23. Seeley, R.T.: In: CIME Conference on Pseudo-Differential Operators. Stresa (1968)

24. Segal, G.B.: Fredholm complexes. Quart. J. Math. 21, 385-402 (1970)

25. Segal, G.B.: The definition of conformal field theory. Preprint

26. Segal, G.B.: Geometric aspects of quantum field theory. Proc. Int. Cong. Math. Tokyo, 1990

27. Simon, B.: Trace Ideals and their Applications. London Mathematical Society Lecture Notes Vol. 35, Cambridge: Camb. Univ. Press, 1979

28. Witten, E.: Quantum field theory, Grassmannians and strings. Commun. Math. Phys. 113, 529-600 (1988)

29. Woodhouse, N.M.J.: Geometric Quantization. Oxford: Oxford Univ. Press, 1992

30. Wojciechowski, K.P.: Smooth, self-adjoint Grassmanian, the $\eta$-invariant, and $\zeta$-determinant of boundary problems. IUPUI Preprint (1995) 